\title{
Envelope model for passive magnetic focusing of an intense proton or ion beam propagating through thin foils
}

\author{
Steven M. Lund* and Ronald H. Cohen ${ }^{\dagger}$ \\ Lawrence Livermore National Laboratory, Livermore, California 94550, USA
}

Pavel A. $\mathrm{Ni}^{\ddagger}$

Lawrence Berkeley National Laboratory, Berkeley, California 94720, USA

(Received 20 January 2012; revised manuscript received 27 March 2013; published 23 April 2013)

Ion beams (including protons) with low emittance and high space-charge intensity can be propagated with normal incidence through a sequence of thin metallic foils separated by vacuum gaps of order the characteristic transverse beam extent to transport/collimate the beam or to focus it to a small transverse spot. Energetic ions have sufficient range to pass through a significant number of thin foils with little energy loss or scattering. The foils reduce the (defocusing) radial electric self-field of the beam while not altering the (focusing) azimuthal magnetic self-field of the beam, thereby allowing passive self-beam focusing if the magnetic field is sufficiently strong relative to the residual electric field. Here we present an envelope model developed to predict the strength of this passive (beam generated) focusing effect under a number of simplifying assumptions including relatively long pulse duration. The envelope model provides a simple criterion for the necessary foil spacing for net focusing and clearly illustrates system focusing properties for either beam collimation (such as injecting a laser-produced proton beam into an accelerator) or for magnetic pinch focusing to a small transverse spot (for beam driven heating of materials). An illustrative example is worked for an idealization of a recently performed laser-produced proton-beam experiment to provide guidance on possible beam focusing and collimation systems. It is found that foils spaced on the order of the characteristic transverse beam size desired can be employed and that envelope divergence of the initial beam entering the foil lens must be suppressed to limit the total number of foils required to practical values for pinch focusing. Relatively modest proton-beam current at $10 \mathrm{MeV}$ kinetic energy can clearly demonstrate strong magnetic pinch focusing achieving a transverse rms extent similar to the foil spacing (20-50 $\mu \mathrm{m}$ gaps) in beam propagation distances of tens of $\mathrm{mm}$. This is a surprisingly optimistic result since placing many foils per characteristic beam radius, which one might expect to be necessary to strongly attenuate the self-electric field, would likely result in excessive scattering and loss of focusing from the current neutralization due to the beam propagating too far through solid metal. Results from the envelope model are compared with particle-in-cell simulations to help clarify limits related to envelope-model idealizations. Possible degradations of focusing in situations where strong halo can be generated and where pulse duration is short are clarified.

DOI: 10.1103/PhysRevSTAB.16.044202

PACS numbers: 29.27.Bd, 41.75.-i, 52.59.Sa

\section{INTRODUCTION}

Focusing of space-charge dominated proton and ion beams is required in various applications, including intense beam injectors derived from short pulse laser-illuminated foils via target normal sheath acceleration (TNSA), radiography, generation of beam-heated warm dense matter, proton and ion-based fast ignition concepts, and medical therapies [1-6]. Beams produced by TNSA processes have

\footnotetext{
*smlund@1lnl.gov

†rohen@1lnl.gov

pani@lbl.gov
}

Published by the American Physical Society under the terms of the Creative Commons Attribution 3.0 License. Further distribution of this work must maintain attribution to the author(s) and the published article's title, journal citation, and DOI. comoving electrons which must be stripped while focusing is provided if the beam is injected into a conventional beam transport lattice for further acceleration and phase-space manipulations. Such "collimation" procedures should be simple and compact to enable future TNSA injectors for a wide range of applications. These classes of focusing and collimation applications are a substantial challenge due to strong space-charge forces in the intense beams. To date, active focusing techniques based on both applied solenoid and quadrupole magnets and rf systems have been employed in beam focusing and collimation systems $[7,8]$. Such applied-focusing based systems have critical disadvantages including high beam losses due to chromatic aberrations; often, small focal spot radii $(\$ 50 \mu \mathrm{m})$ are desired, requiring large and expensive magnet systems.

We analyze a structure in which net beam focusing can be passively generated by the beam's own self-magnetic 
field with no external fields applied. As an ion beam propagates in vacuum it interacts with its own selfgenerated defocusing radial electric field and focusing azimuthal magnetic field. In vacuum, the electric defocusing force is always stronger than the magnetic focusing force, resulting in a net transverse expansion of the beam. If the electrical force can be attenuated to the point where it is weaker than the magnetic force by adding conducting surfaces to induce image charges which cancel a sufficient fraction of the beam self-field in the absence of the conductors, then net focusing can occur. The approach examined here is to attenuate the radial electric field by allowing the beam to propagate through a stack of thin metallic foils separated by finite vacuum gaps. Because the tangential electric field but not the tangential magnetic field vanishes at the surface of a good conductor, the metal foils will serve to suppress the electric field in the vacuum gaps while leaving the magnetic field unaffected relative to the value with no foils. This is schematically illustrated in Fig. 1 where a shaped, laser-illuminated foil produces a proton beam by TNSA processes. The beam is then collimated (comoving electrons blocked) and focused by a stack of thin foils. As the beam fills the gap between foils, image charges build up on the surfaces of the foils and generate an image electric field that counteracts the vacuum electric field of the beam in the absence of the foils. The thinness of the foils suppresses eddy current effects while the foils can simultaneously be thick enough to stop electrons with much lower range than protons or ions to suppress undesired "e-cloud" effects. The smaller the gap size, the weaker the net electric field which interacts with the beam, whereas the thin foils do not significantly alter the beam current and the magnetic field produced by the beam remains at its vacuum value. One expects that the gap must be on the order of the characteristic transverse beam extent to significantly attenuate the transverse electric field. Thus, in focusing applications for small transverse beam extent (possibly tens of micron radius), foil stacks with very small gaps may be necessary. However, the foils must have sufficient thickness to provide adequate material strength, to be consistent with fabrication limits, and to be

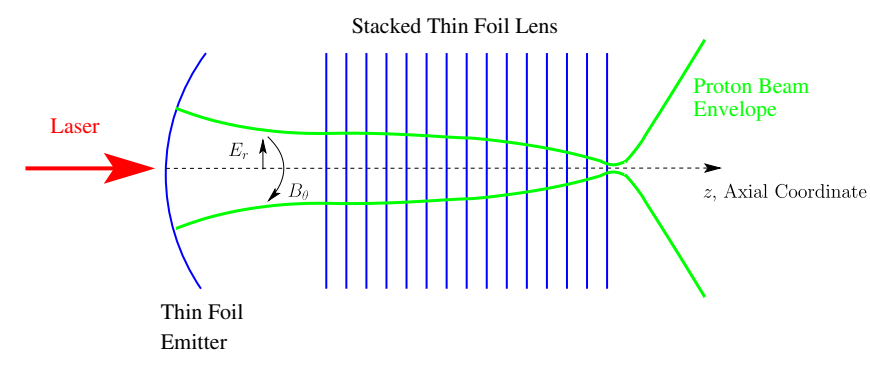

FIG. 1. Schematic of a thin-foil lens focusing a proton beam generated off the back side of a shaped, thin foil illuminated by an intense laser pulse. The beam-generated field azimuthal magnetic field $B_{\theta}$ is focusing while the radial electric field $E_{r}$ is defocusing. able to stop electrons. Therefore, smaller gaps separating the foils also result in the beams propagating more distance through solid metal which can eventually lead to significant reductions in beam energy and defocusing from scattering. The fundamental question is how large a gap can be employed to sufficiently attenuate the electric field while mitigating such deleterious effects.

There are other practical constraints on the foils beyond the issues outlined above. The radial extent of the foils should be much larger than the characteristic transverse beam radius. Although foil lenses can be single shot optics for many applications, the foils must at least survive heating over the duration of the beam pulse from whatever fraction of the beam energy is deposited in the foil, as well as heating from electrons stopped in the foils. In addition to limits imposed by mechanical strength needs and fabrication, the minimal foil thickness can also be limited by the value necessary to stop energetic electrons (at least in the first foil) which can be present from the injected beam (e.g., beams produced by short pulse lasers in TNSA applications) as well as "knock-on" electrons produced by beam particles incident on the foils due to a Rutherford scattering mechanism (production cross sections scale with beam ion atomic number $Z_{b}$ and the foil conductor atomic number $Z_{\text {foil }}$ as $Z_{b}^{2} Z_{\text {foil }}^{2}$ making the issue much less for protons than heavier ions) [9]. Aluminum is an obvious choice for foil material since among good conductors it is light (high beam range leading to less foil heating), low atomic number $Z_{\text {foil }}$ (less knock-on electrons), and has favorable material properties for fabrication. Fewer knock-on electrons diminishes the potential for partial neutralization of the beam current which could attenuate the magnetic focusing force. Another option is carbon which is mechanically more robust but has worse conductivity and higher energy loss. Because the main purpose of the foils is to attenuate the radial electric field, alignment tolerances (tilts, offsets, distortions) are not anticipated to be demanding insofar as neighboring foils do not touch. Chemical etching processes have recently been demonstrated at TU-Darmstadt $[10,11]$ to fabricate stacks of $\gtrsim 1 \mu \mathrm{m}$ thick Al foils separated by gaps with $\gtrsim 1 \mu \mathrm{m}$ separating adjacent foils with $\lesssim 10 \mathrm{~mm}$ characteristic transverse extent. Many (hundreds to thousands) such foils can be stacked with the necessary precision and stability and spacing between adjacent foils can be variable. It is also possible that a regular or irregular pattern of small holes can be placed in foils to produce a mesh with a higher ion transparency than a solid foil while not significantly altering the electric-field attenuation relative to a solid foil. Similarly, a lower density metal foam or a regular or semi-irregular arrangement of fine wires could be used. However, such a mesh may result in a detrimental partial current neutralization because it may allow a significant fraction of beam-generated electrons to pass through the mesh. Alternatively, materials with strongly anisotropic 
conductivity could be considered. Practical issues associated with the choice of foil parameters are analyzed in more detail in Ref. [12]. The purpose of the present article is primarily to systematically analyze the basic focusing properties of the system.

Needed foil properties depend on details of the specific application. For present-generation TNSA produced proton beams there may be significant energy spread and short pulse duration [10,13-16]. For an illustrative example, we provide estimates based on an idealized TNSA protonbeam experiment analyzed in later sections with $\sim 400 \mathrm{~A}$ of protons in a 4 ps pulse window about $10 \mathrm{MeV}$ and a broad energy spectrum and $\sim 50 \mu \mathrm{m}$ spaced $\mathrm{Al}$ foils that are $\sim 0.5 \mu \mathrm{m}$ thick [10]. More details are provided in Ref. [12]. In this case, the total proton energy deposited over the full pulse is sufficient to evaporate several thin foils. However, most of the deposited energy comes from protons with energies less than, or of order of, the mean beam energy (1-5 MeV). Hence, if the higher-energy portion of the proton beam $(\sim 10 \mathrm{MeV})$ can be focused, one can perform a foil transport experiment using this energy group, before the more numerous lower-energy protons arrive at the foils and damage them. Estimates indicate that foils as thin as $0.5 \mu \mathrm{m}$ are adequate to be structurally stable, survive heating during the $\sim 10 \mathrm{MeV}$ portion of the pulse, and stop comoving electrons (few hundred $\mathrm{keV}$ ). Scattering is expected to contribute a small angular beam divergence at each foil which can be part mitigated by keeping the foils thin. In this idealized theoretical study centered on infinitesimally thin foils, scattering is neglected. The influence of finite scattering on focusing properties (including influence on achievable spot size) is being investigated and will be submitted for publication [17]. A classical Drude-type model [18] can be applied to estimate image charge response time from the plasma frequency associated with conduction electrons in the $\mathrm{Al}$ metal foils. This gives a $\sim 0.0003$ ps response time which is much more rapid than the 4 ps pulse duration and the $\sim 1$ ps transit time of a nominal $10 \mathrm{MeV}$ particle between the foils. Light propagation time across a $50 \mu \mathrm{m}$ foil gap is of order $0.2 \mathrm{ps}$ which suggests small electromagnetic effects.

The idea to utilize thin, conducting foils to passively focus a particle beam has been analyzed and experimentally demonstrated for electrons [19-21]. For electrons, semitransparent metal meshes are employed in place of foils. Numerous schemes to focus ion beams using fine conducting wires, meshes, and other structures to attenuate the defocusing self-electric field and/or utilizing plasma generated fields to neutralize or overwhelm defocusing beam self-field components have been proposed. Many of these were associated with studies in collective ion acceleration [22] and documentation can be difficult to obtain. Recently, foil focusing for heavy ions and protons using the method outlined above has been proposed by Logan [23] to focus a heavy ion beam to a small spot for the proposed X-target [6] for heavy-ion fusion and various fast ignition and warm dense matter applications. One of the motivations of the present study is to derive a simple and efficient theoretical model which can be applied to guide experiments to verify passive foil focusing for both protons and heavy ions. This should aid both carrying out higher detail simulations and design of proof-of-principle experiments to enable applications in both ion and proton beam focusing to a small spot for targets and for beam collimation for injection in an accelerator. Short pulse laser-illuminated foils produce proton beams with very low emittances, high enough energy, and high spacecharge intensity [24-27]. Such proton beams provide an opportunity to test passive foil focusing on existing laboratory systems. However, present systems typically also have short pulse duration and large distribution spreads which may need to be controlled for clear demonstration of passive magnetic focusing. Electric-field attenuation predicted by the theory could also be experimentally tested on intense, low-energy proton and ion beams propagating through closely spaced semitransparent meshes.

An envelope model is derived under a set of simplifying assumptions: the beam pulse is axially long relative to the gap between foils and the spread in beam axial energy is negligible. It is shown that the self-magnetic-focusing term is independent of the radial charge distribution provided the beam density profile varies slowly in the axial coordinate. The self-electric defocusing force is calculated employing a Green's function and is explicitly evaluated for self-similarly evolving density profiles which evolve only weakly in the axial coordinate between pairs of foils. Little difference is found in the form of the defocusing force for Gaussian and uniform radial charge-density profiles and similarly small differences are expected for other reasonably smooth radial charge profiles. Provisions are included in the model for augmented focusing by a superimposed solenoidal field which can provide additional system flexibility for tuning. Emittance measures are carefully analyzed to include in the proper form when solenoidal focusing is employed. Terms in the envelope model clearly illustrate where the magnetic focusing force is larger than the electric defocusing force and the strength of the effect. The model enables rapid evaluation of maximum gap size between the foils to produce a small transverse beam spot for magnetic pinch focusing or to maintain transverse beam size for beam collimation and transport. An idealization of a recently performed short pulse laser driven TNSA produced proton-beam experiment at the PHELIX facility at GSI [10] is analyzed for an illustrative application of the model. This experiment will be reported separately. Results for a long pulse $10 \mathrm{MeV}, 400 \mathrm{~A}$ proton beam with initial transverse rms width $\left\langle x^{2}\right\rangle_{\perp}^{1 / 2} \sim 200 \mu \mathrm{m}$ shows that several hundreds of $\sim 0.5 \mu \mathrm{m}$ thick foils separated by 20-50 $\mu \mathrm{m}$ gaps should produce a strong, observable focusing effect for a viable proof-of-principle experiment provided initial beam divergence can be limited. The envelope model helps clarify a critical sensitivity to initial 
beam divergence for viable experiments. Particle-in-cell (PIC) simulations are applied with reference to this example to help elucidate where envelope-model restrictions may break down due to model idealizations including evolution of the distribution and halo, short pulse duration, and energy spread. It is found that short pulse duration increases the focal distance when the initial beam length becomes comparable to its radius, but does not appreciably affect the minimum spot size, and the radial particle distribution evolves from the initially loaded shape in such a way as to degrade (increase) somewhat the minimum spot size. Regarding energy spread, simply spreading the particles in a finite-length monoenergetic beam over a finite energy window degrades focusing due to beam spreading. On the other hand, in the idealized modeling described above, the current in the monoenergetic beam was chosen to be about that in a $1 \mathrm{MeV}$ window of a much broader energy distribution. Simulations with such an extended energy distribution (with a correspondingly larger total current) show enhanced focusing relative to the corresponding monoenergetic case, because of the magnetic field contribution due to neighboring energy groups.

The organization of this paper is the following. In Sec. II we overview the idealized geometry (Sec. II A) and simplifying model assumptions (Sec. II B). Self-fields produced by the system consistent with the presence of the conducting foils are evaluated in Sec. III for both magnetic (Sec. III A) and electric (Sec. III B) components. These results are used in Sec. IV to derive a statistical envelope equation describing the evolution of the transverse beam size. In this section, approximate transverse particle trajectory equations are derived (Sec. IVA), which are then averaged over the beam (Sec. IV B) to obtain reduced model envelope equations. The envelope model predicts (Sec. IV C) when self-magnetic focusing forces can overcome the electric defocusing force attenuated by the foils to allow passive focusing and can be applied to predict the achievable beam spot in magnetic pinch focusing. An illustrative application of the envelope model in Sec. V demonstrates the utility of the envelope model in understanding beam evolution in the thin-foil lens. Particle-incell simulations presented in Sec. VI are carried out under a lesser degree of idealization and help quantify where the envelope model can be trusted and deviations which might be reasonably expected. Concluding discussions in Sec. VII frame results presented.

\section{GEOMETRY AND BEAM MODEL}

The geometry of the foil system is specified (Sec. II A) and modeling idealizations of the beam are outlined (Sec. II B).

\section{A. Geometry}

The idealized geometry of the thin-foil transport lattice is illustrated in Fig. 2. A beam of particles of charge $q$ and

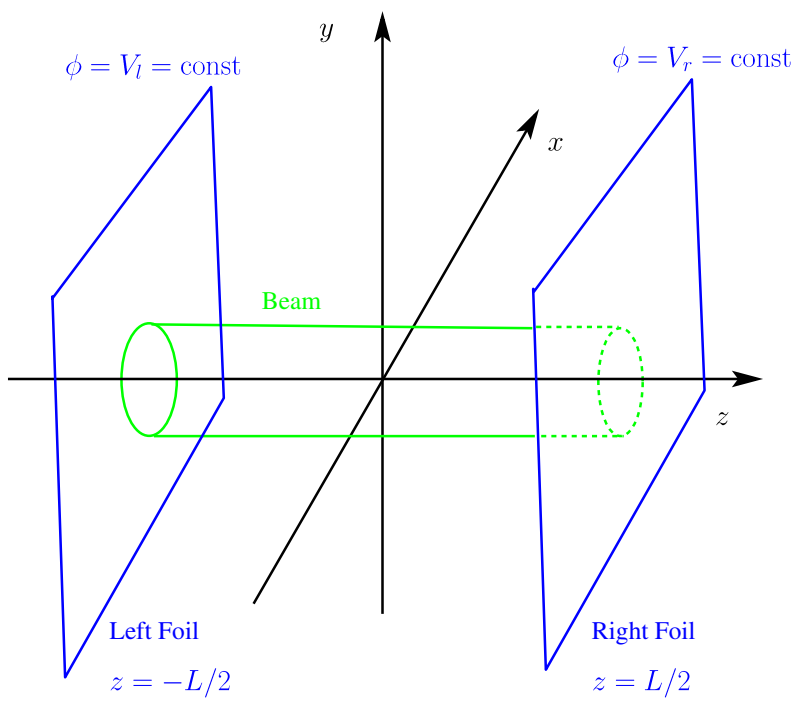

FIG. 2. Axisymmetric beam between two, thin conducting foils located at $z= \pm L / 2$. The foils are biased to potentials $\phi=V_{l}$ and $\phi=V_{r}$ on the left and right.

mass $m$ propagates in the axial $z$ direction. A sequence of thin, equally spaced and perfectly conducting foils are located at $z=$ const planes spaced an axial distance $L$ apart. We analyze the region between a pair of foils located at $z=-L / 2$ and $z=L / 2$ which, for generality, are assumed biased to potential $\phi=V_{l}=$ const and $\phi=V_{r}=$ const. (In later modeling we take $V_{l}=V_{r}=0$, but it is useful to retain these biases to apply results in later applications where they might be retained for electron sweeping.) The particles are assumed to be sufficiently energetic that the beam can penetrate many thin foils with negligible loss in axial kinetic energy and negligible transverse scattering. It is further assumed that the beam is both sufficiently long to fill the axial space between several successive foils and is long compared to its characteristic transverse radius, and is nearly monoenergetic with axial velocity $V_{b}=\beta_{b} c=$ const. Here, $\beta_{b}$ is the axial relativistic factor of the beam and $c$ is the speed of light in vacuo. The axial velocity $V_{b}=\beta_{b} c$ is related to the axial kinetic energy $\mathcal{E}_{b}$ of the particles by $\mathcal{E}_{b}=\left(\gamma_{b}-1\right) m c^{2}$ with $\gamma_{b}=1 / \sqrt{1-\beta_{b}^{2}}$. For possible augmented beam focusing and system tunability, we allow for a superimposed applied solenoidal magnetic field $\mathbf{B}^{a}$ with

$$
\mathbf{B}^{a}=-\frac{1}{2} \frac{\partial B_{z 0}(z)}{\partial z}(\hat{\mathbf{x}} x+\hat{\mathbf{y}} y)+\hat{\mathbf{z}} B_{z 0}(z)
$$

Here, $B_{z 0}(z)=B_{z}^{a}\left(\mathbf{x}_{\perp}=0, z\right)$ is the specified on-axis magnetic field of the solenoid. The solenoidal field can provide additional beam focusing consistent with maintaining an axisymmetric $(\partial / \partial \theta=0)$ beam. The expression in Eq. (1) allows axial fringe-field variation in $B_{z}^{a}$ while retaining terms consistent with linear beam optics [28,29]. 


\section{B. Beam model}

We adapt a Vlasov model and the single-particle distribution function $f_{b}(\mathbf{x}, \mathbf{p}, z)$ describing the beam in $\mathbf{x}$-p coordinate-momentum phase space with the axial coordinate $z$ used as the independent variable. The beam distribution $f_{b}$ is constrained as follows. The beam charge density $\rho_{b}=q \int d^{3} p f_{b}$ is axisymmetric $(\partial / \partial \theta=0)$ and fills the region between any two sets of foils with negligible variation in $z$, i.e., $\rho_{b}=\rho_{b}(r)$ with $r=\sqrt{x^{2}+y^{2}}$. We take the total charge density of the system to be

$$
\rho \simeq\left(1-\mathcal{F}_{\rho}\right) \rho_{b}(r) .
$$

Here, $\mathcal{F}_{\rho}=$ const $\in[0,1]$ is a specified neutralization fraction to allow for low-order modeling of partial neutralization of the beam charge density by electrons which might, for some choices of system parameters, be emitted from or penetrate the foils. The axial beam velocity $V_{b}=$ $\beta_{b} c=$ const further constrains the beam distribution through $V_{b}=\int d^{3} p v_{z} f_{b} / \int d^{3} p f_{b}$ with $v_{z}$ the axial particle velocity. The beam velocity $V_{b}=\beta_{b} c$ is set consistently with the axial particle energy $\mathcal{E}_{b}=\left(\gamma_{b}-1\right) m c^{2}$. The beam line charge

$$
\lambda=2 \pi \int_{0}^{\infty} d r r \rho_{b}(r)=\text { const }
$$

or equivalently the beam current $I=\beta_{b} c \lambda$, is regarded as specified. The beam current density is consistently set by $\mathbf{J}_{b} \simeq \hat{\mathbf{z}} J_{b z}$ with $J_{b z}=q \int d^{3} p v_{z} f_{b}=\beta_{b} c \rho_{b}$. We take the total current density of the system to be

$$
\mathbf{J} \simeq \hat{\mathbf{z}}\left(1-\mathcal{F}_{J}\right) \beta_{b} c \rho_{b}(r) .
$$

Here, $\mathcal{F}_{J}=$ const $\in[0,1]$ is a specified neutralization fraction to allow for low-order modeling of partial neutralization of the beam current density by electrons; in general $\mathcal{F}_{J} \neq \mathcal{F}_{\rho}$. Scattering of ions by interactions with other ions, electrons, material in the foils, or neutral species are all neglected.

We define a transverse $(\perp)$ statistical average over the beam distribution $f_{b}(\mathbf{x}, \mathbf{p}, z)$ by

$$
\langle\cdots\rangle_{\perp} \equiv \frac{\int d^{2} x_{\perp} \int d^{3} p \cdots f_{b}}{\int d^{2} x_{\perp} \int d^{3} p f_{b}}
$$

where $\mathbf{x}_{\perp}=\hat{\mathbf{x}} x+\hat{\mathbf{y}} y$ denotes the transverse coordinate. In such averages, we take the functional form of the beam charge density $\rho_{b}(r)=q \int d^{3} p f_{b}$ to be fixed. However, transverse statistical averages are allowed to evolve in $z$ due to characteristic scale lengths in the functional form of $\rho_{b}$ evolving. This evolution is consistent with conservation of system line charge $\lambda=$ const under the influence of focusing self-magnetic fields and defocusing self-electric fields generated by the beam and electrons in the presence of the foils as well as any applied magnetic field $\mathbf{B}^{a}$. Following an evaluation of system self-fields in Sec. III, statistical envelope equations for the transverse rms-beam

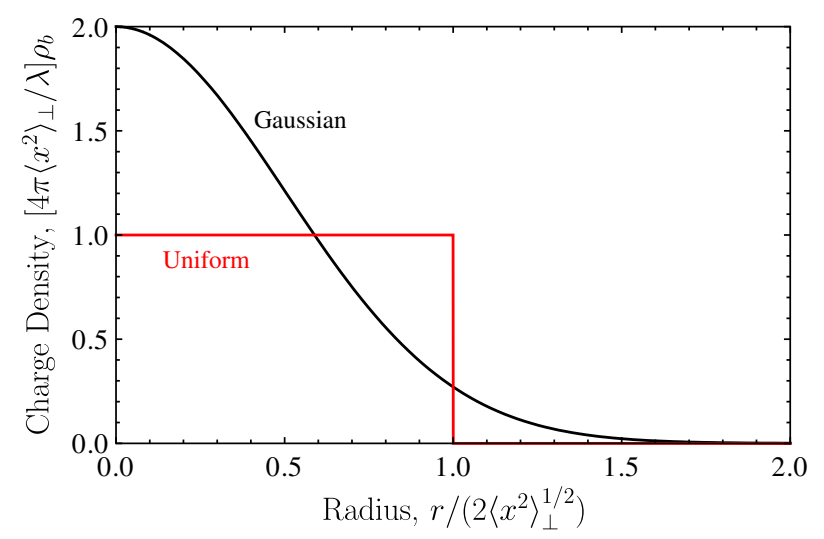

FIG. 3. Scaled radial beam charge density $\rho_{b}(r)$ [Eq. (6)] versus scaled radius $r$ for Gaussian (black) and uniform (red) beams. Shown for rms-equivalent profiles with $\left\langle x^{2}\right\rangle_{\perp}^{1 / 2}=$ $\sigma_{x}=r_{b} / 2$.

width $\left\langle x^{2}\right\rangle_{\perp}^{1 / 2}$ are derived in Sec. IV. Two forms of $\rho_{b}$ are analyzed corresponding to Gaussian- and uniform-chargedensity beams with

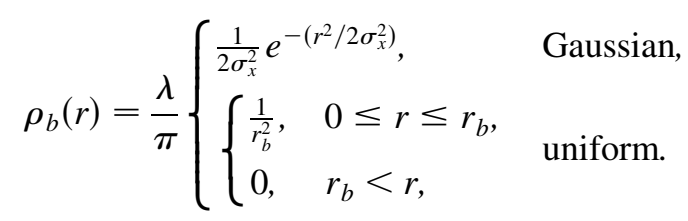

Here, $\sigma_{x}=\left\langle x^{2}\right\rangle_{\perp}^{1 / 2}$ is the rms width in $x$ of the Gaussian beam and $r_{b}=2\left\langle x^{2}\right\rangle_{\perp}^{1 / 2}$ is the edge radius of the uniform beam. Both $\sigma_{x}$ and $r_{b}$ are allowed to evolve in $z$. The choice of Gaussian $\rho_{b}$ may be appropriate for a neutralized intense beam near a laser-plasma source where a short pulse laser beam typically has a Gaussian-distributed focal spot and the expanding plasma sheath appears to produce an approximately Gaussian beam [30,31]. Conversely, the choice of uniform $\rho_{b}$ may be appropriate for an unneutralized intense beam emerging from a linear transport channel where Debye screening of the linear applied-focusing force leads to an approximately uniform charge-density beam $[28,32,33]$. The two density profiles are contrasted in Fig. 3, where $\rho_{b}$ is plotted versus $r$ for "rms-equivalent" profiles with equal line charge $\lambda$ and rms width $\left\langle x^{2}\right\rangle_{\perp}^{1 / 2}$. This rms equivalency requires that $r_{b}=2 \sigma_{x}$.

\section{SELF-FIELD SOLUTION}

Magnetic (Sec. III A) and electric (Sec. III B) self-fields produced by the beam and neutralizing electrons are calculated under a quasistatic approximation consistent with the presence of thin metallic foils. We assume that the magnetic field is unaffected by the foils while the tangential electric field at the foil surfaces is reduced to near zero. In the physical situation with small but finite-thickness foils, this is equivalent to assuming good but not perfect conductivity, so that the (azimuthal) magnetic field can 
penetrate through finite-thickness foils during the beam's transit, while the electric field remains small within the foils [34]. Longer beam pulses with relatively slow variation in self-fields generated should be more accurately represented by this idealization. Fields are explicitly evaluated for the Gaussian- and the uniform-chargedensity beam models presented in Sec. II B. Results are checked against limiting forms amenable to simpler analytic solution.

\section{A. Magnetic field}

We take the magnetic field $\mathbf{B}$ produced by the beam and electrons to be related to a self-field vector potential A by $\mathbf{B}=\boldsymbol{\nabla} \times \mathbf{A}$. Adopting the Coulomb gauge choice $\boldsymbol{\nabla} \cdot \mathbf{A}=0$, the Maxwell equations describing the selfmagnetic field in a quasistatic approximation can be expressed as [34]

$$
\nabla^{2} \mathbf{A}=-\mu_{0} \mathbf{J}
$$

Here, $\mu_{0}=4 \pi \times 10^{-7}$ Henry $/ \mathrm{m}$ is the permeability of free space. Taking $\mathbf{J}$ to be given by Eq. (4) and consistently taking $\mathbf{A} \simeq \hat{\mathbf{z}} A_{z}$, Eq. (7) is approximated by

$$
\nabla^{2} A_{z} \simeq-\left(1-\mathcal{F}_{J}\right) \mu_{0} \beta_{b} c \rho_{b} .
$$

Because the thin foils do not influence the quasistatic magnetic field, Eq. (8) is the same form as the vacuum Poisson equation for the electrostatic potential $\phi_{v}$ produced by the unneutralized beam in the absence of the foils (free space), i.e.,

$$
\nabla^{2} \phi_{v}=-\frac{\rho_{b}}{\epsilon_{0}}
$$

Here, $\epsilon_{0}$ is the permittivity of free space and $\mu_{0} \epsilon_{0}=1 / c^{2}$. Comparing Eqs. (8) and (9), we have

$$
A_{z} \simeq\left(1-\mathcal{F}_{J}\right) \frac{\beta_{b}}{c} \phi_{v}
$$

and the magnetic self-field $\mathbf{B}=\boldsymbol{\nabla} \times \mathbf{A}$ is then

$$
\mathbf{B} \simeq \hat{\boldsymbol{\theta}} B_{\theta} \simeq-\hat{\boldsymbol{\theta}}\left(1-\mathcal{F}_{J}\right) \frac{\beta_{b}}{c} \frac{\partial \phi_{v}}{\partial r} .
$$

Integrating Eq. (9) once with respect to $r$ and substituting into Eq. (11) then gives

$$
B_{\theta} \simeq\left(1-\mathcal{F}_{J}\right) \frac{\beta_{b}}{\epsilon_{0} c r} \int_{0}^{r} d \tilde{r} \tilde{r} \rho_{b}(\tilde{r}) .
$$

For the case of Gaussian- and uniform-charge-density beams described by Eqs. (6) and (12) for $B_{\theta}$ can be explicitly evaluated to show that

$$
B_{\theta} \simeq\left(1-\mathcal{F}_{J}\right) \frac{\lambda \beta_{b}}{2 \pi \epsilon_{0} c} \begin{cases}(1 / r)\left[1-e^{-r^{2} /\left(2 \sigma_{x}^{2}\right)}\right], & \text { Gaussian, } \\ \begin{cases}r / r_{b}^{2}, & 0 \leq r \leq r_{b},\end{cases} & \text { uniform. }\end{cases}
$$

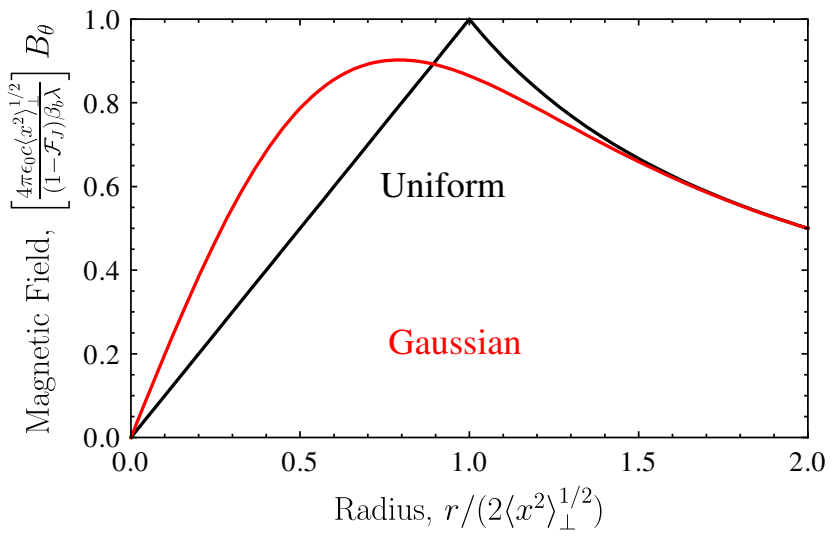

FIG. 4. Scaled self-magnetic field $B_{\theta}$ [Eq. (13)] versus scaled radial coordinate $r$ for beams with Gaussian (black) and uniform (red) charge density. Shown for rms-equivalent distributions with $\left\langle x^{2}\right\rangle_{\perp}^{1 / 2}=\sigma_{x}=r_{b} / 2$.

These fields are plotted in Fig. 4 for rms-equivalent beams (equal $\lambda$ and rms-beam radii) with $r_{b}=2 \sigma_{x}$. For the uniform-density beam, the field is linear within $\left(r \leq r_{b}\right)$ the beam and falls off as $1 / r$ outside $\left(r_{b} \leq r\right)$ the beam. For the Gaussian beam, there is no sharp beam edge, but the field is approximately linear for small $r$ and increases more rapidly for small $r$ than the uniform case due to the equivalent beam density being higher for the Gaussian case and approximately uniform in the core. For radii well outside the core of the Gaussian beam $\left(r \gg \sigma_{x}\right)$, the field asymptotes with the same $\sim 1 / r$ variation as for the uniform beam.

\section{B. Electric field}

We model the self-electric field $\mathbf{E}$ produced by the beam and electrons as electrostatic and take $\mathbf{E}=-\nabla \phi$ where the potential $\phi$ satisfies the Poisson equation

$$
\nabla^{2} \phi=-\frac{\rho}{\epsilon_{0}}
$$

between two foils held at potentials $\phi=V_{l}=$ const at $z=$ $-L / 2$ (left foil) and $\phi=V_{r}=$ const at $z=L / 2$ (right foil) as sketched in Fig. 2. Using linear superposition, $\phi$ can be expressed as

$$
\phi=\frac{1}{2}\left(V_{r}+V_{l}\right)+\left(V_{r}-V_{l}\right) \frac{z}{L}+\left(1-\mathcal{F}_{\rho}\right) \phi_{g}
$$

where $\phi_{g}$ is the solution to

$$
\nabla^{2} \phi_{g}=-\frac{\rho_{b}}{\epsilon_{0}}
$$

subject to $\phi_{g}=0$ on the foils. 
Equation (16) for $\phi_{g}$ can be formally solved as [34]

$$
\phi_{g}=\frac{1}{4 \pi \epsilon_{0}} \int_{-L / 2}^{L / 2} d \tilde{z} \int d^{2} \tilde{x}_{\perp} \rho_{b}(\tilde{\mathbf{x}}) G(\mathbf{x}, \tilde{\mathbf{x}}),
$$

where $G(\mathbf{x}, \tilde{\mathbf{x}})$ is the Dirichlet Green's function satisfying

$$
\nabla^{2} G(\mathbf{x}, \tilde{\mathbf{x}})=-4 \pi \delta(\mathbf{x}-\tilde{\mathbf{x}})
$$

with $\quad G(\mathbf{x}, \tilde{\mathbf{x}})=G(\tilde{\mathbf{x}}, \mathbf{x})$ and $\left.\quad G(\mathbf{x}, \tilde{\mathbf{x}})\right|_{z= \pm L / 2}=0$. In Eq. (18) $\delta(\mathbf{x})=\delta(x) \delta(y) \delta(z)$, where $\delta(x)$ represents the usual Dirac-delta function in one dimension. An explicit representation for $G$ can be calculated in a convenient expanded form as [34]

$$
\begin{aligned}
G(\mathbf{x}, \tilde{\mathbf{x}})= & 2 \sum_{n=-\infty}^{\infty} \int_{0}^{\infty} d k e^{i n(\theta-\tilde{\theta})} J_{n}(k r) J_{n}(k \tilde{r}) \\
& \times \frac{\sinh \left[k\left(L / 2+z_{<}\right)\right] \sinh \left[k\left(L / 2-z_{>}\right)\right]}{\sinh (k L)} .
\end{aligned}
$$

Here, $i \equiv \sqrt{-1}, \theta=\tan ^{-1}(y, x)$ is the azimuthal angle, $z_{>} / z_{<}$denotes the greater/lesser of $z$ and $\tilde{z}, J_{n}(x)$ is an $n$th order ordinary Bessel function [35], and $\sinh (x)$ is the hyperbolic sine function. Using Eq. (19) in Eq. (17), we obtain

$$
\begin{aligned}
\phi_{g}= & \frac{1}{\epsilon_{0}} \int_{0}^{\infty} \frac{d k}{k} \frac{\cosh (k L / 2)-\cosh (k z)}{\cosh (k L / 2)} J_{0}(k r) \\
& \times \int_{0}^{\infty} d \tilde{r} \tilde{r} \rho_{b}(\tilde{r}) J_{0}(k \tilde{r}),
\end{aligned}
$$

where $\cosh (x)$ is the hyperbolic cosine function. The radial and axial contributions to the electric-field components can be calculated as

$$
\begin{aligned}
-\frac{\partial \phi_{g}}{\partial r}= & \frac{1}{\epsilon_{0}} \int_{0}^{\infty} d k \frac{\cosh (k L / 2)-\cosh (k z)}{\cosh (k L / 2)} J_{1}(k r) \\
& \times \int_{0}^{\infty} d \tilde{r} \tilde{r} \rho_{b}(\tilde{r}) J_{0}(k \tilde{r}), \\
-\frac{\partial \phi_{g}}{\partial z}= & \frac{1}{\epsilon_{0}} \int_{0}^{\infty} d k \frac{\sinh (k z)}{\cosh (k L / 2)} J_{0}(k r) \\
& \times \int_{0}^{\infty} d \tilde{r} \tilde{r} \rho_{b}(\tilde{r}) J_{0}(k \tilde{r}) .
\end{aligned}
$$

Limiting forms of the field solution are useful for both consistency checks of the Green's function based expressions in Eqs. (20) and (21) and for use in a scaling analysis carried out in Sec. IV. First, for the case of small beam aspect ratio where the characteristic transverse scale of the beam $r_{\perp}$ is small relative to the foil separation $L$, one expects that for $z$ not close to the foils that

$$
\phi_{g} \simeq \phi_{v}
$$

where $\phi_{v}$ is the solution to the vacuum Poisson equation (9). Solving for $\phi_{v}$ then gives the expected limiting form of the radial electric field

$$
-\frac{\partial \phi_{g}}{\partial r} \simeq \frac{\lambda}{2 \pi \epsilon_{0}} \begin{cases}(1 / r)\left[1-e^{-r^{2} /\left(2 \sigma_{x}^{2}\right)}\right], & \text { Gaussian, } \\
\left\{\begin{array}{ll}
r / r_{b}^{2}, & 0 \leq r \leq r_{b}, \\
1 / r, & r_{b} \leq r,
\end{array} \quad\right. \text { uniform, }\end{cases}
$$

for Gaussian and uniform charge-density beams with $\rho_{b}(r)$ given by Eq. (6). Validity of these expressions requires $\sigma_{x} / L \ll 1$ and $r_{b} / L \ll 1$ for the Gaussian and uniform cases. Next, we consider the opposite limit of large beam aspect ratio where the foils are close together relative to the characteristic transverse dimension $r_{\perp}$ of the beam. It is shown in Appendix A using a power-series expansion, that to leading order in $\left(L / r_{\perp}\right)^{2}$ we have

$$
\phi_{g} \simeq \frac{L^{2} \rho_{b}(r)}{8 \epsilon_{0}}\left(1-\frac{4 z^{2}}{L^{2}}\right)
$$

This leading-order solution is consistent with merely neglecting transverse derivatives (i.e., $\frac{\partial}{\partial r}=0$ ) in the Poisson equation (16). The corresponding radial and axial field components are

$$
\begin{aligned}
& -\frac{\partial \phi_{g}}{\partial r} \simeq-\frac{L^{2}}{8 \epsilon_{0}} \frac{\partial \rho_{b}(r)}{\partial r}\left(1-\frac{4 z^{2}}{L^{2}}\right), \\
& -\frac{\partial \phi_{g}}{\partial z} \simeq \frac{\rho_{b}(r)}{\epsilon_{0}} z .
\end{aligned}
$$

From Eq. (25), the $z$-averaged radial electric field between the foils is

$$
\begin{aligned}
-\int_{-L / 2}^{L / 2} \frac{d z}{L} \frac{\partial \phi_{g}}{\partial r} & \simeq-\frac{L^{2}}{12 \epsilon_{0}} \frac{\partial \rho_{b}}{\partial r} \\
& =\frac{\lambda L^{2}}{12 \pi \epsilon_{0}} \begin{cases}\frac{r}{2 \sigma_{x}^{4}} e^{-r^{2} /\left(2 \sigma_{x}^{2}\right)}, & \text { Gaussian, } \\
\frac{1}{r_{b}^{2}} \delta\left(r_{b}-r\right), & \text { uniform, }\end{cases}
\end{aligned}
$$

for Gaussian- and uniform-density beams. Comparison of the result in Eq. (26) for a Gaussian-density beam with Green's function results shows that the expanded form is accurate to within $1 \%$ fractional error within the "core" of the beam (taken to be $0 \leq r / \sigma_{x} \leq 3$ ) for $\sigma_{x} / L>7$. For uniform-density beams, the sharp beam edge complicates accuracy estimates, though the sharp residual field localized near the beam edge modeled by the delta function is consistent with results presented below for the large aspect-ratio radial field using the Green's function expressions. One can refine the uniform-beam result in Eq. (26) by performing a boundary-layer analysis about the beam edge; the result is a finite $z$-averaged radial electric field, with a maximum value of order $L /\left(12 \pi \epsilon_{0} r_{b}^{2}\right)$ in a boundary layer of width of order $L$. (These estimates are consistent with exact results later presented in Fig. 5.)

In Sec. IV, we employ the $z$ average of $\frac{-\partial \phi_{g}}{\partial r}$ between the foils to simplify the envelope model derived. Averaging the Green's function results in Eq. (21) gives 

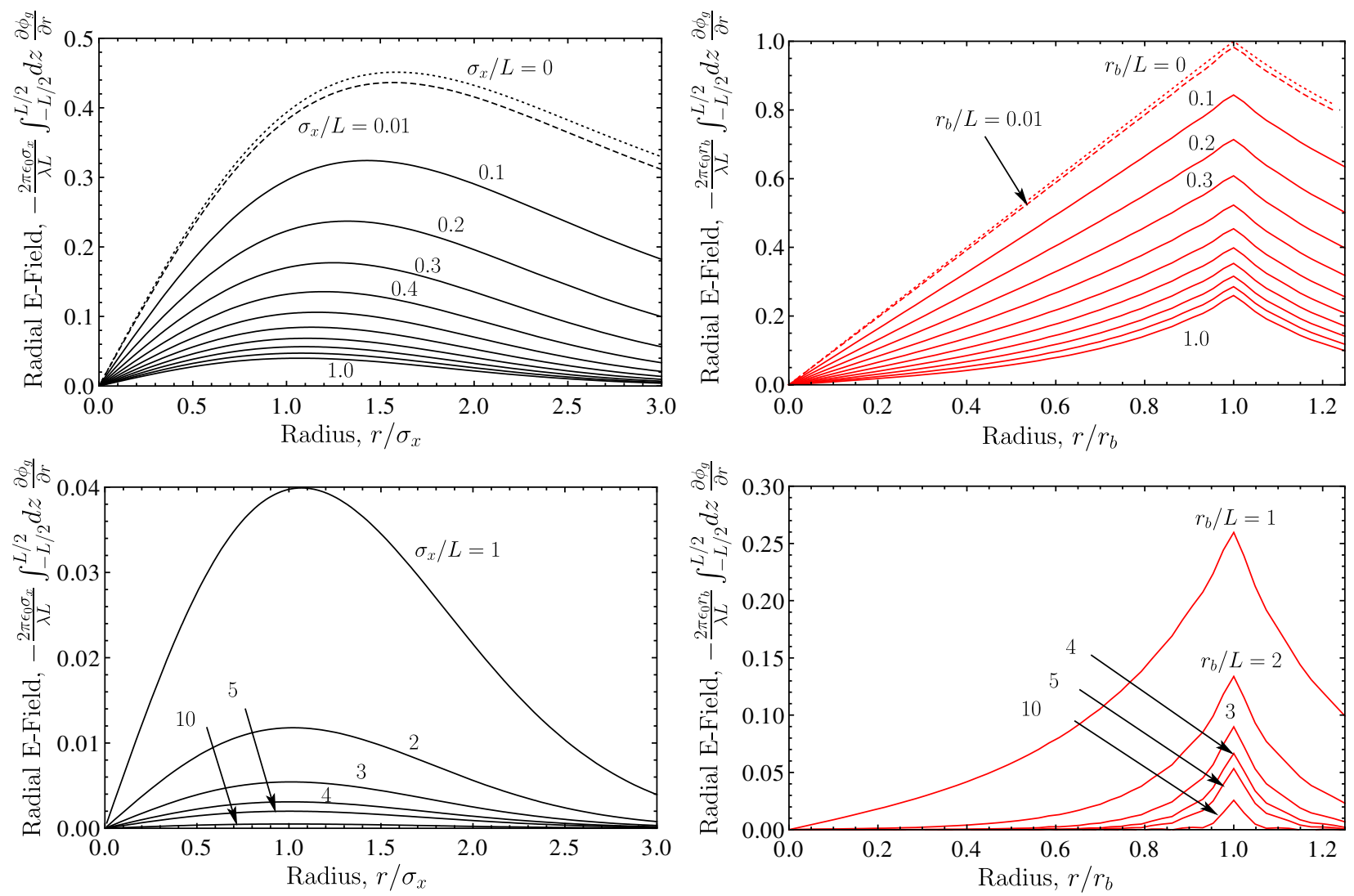

FIG. 5. Plots of scaled $z$-averaged radial electric field $-\int_{-L / 2}^{L / 2} \frac{d z}{L} \frac{\partial \phi_{g}}{\partial r}$ [Eq. (29)] verses scaled radial coordinate $r$ for indicated values of beam aspect ratio. Results shown for Gaussian (black, left column) and uniform (red, right column) density beam profiles with the aspect ratio measured by $\sigma_{x} / L$ and $r_{b} / L$, respectively. Solid curves correspond to uniform increments of aspect ratio and dotted and dashed curves correspond to special values to show limiting and near limiting aspect-ratio curves. Lower row plots show results for large aspect ratio on an expanded scale.

$$
\begin{aligned}
& -\int_{-L / 2}^{L / 2} \frac{d z}{L} \frac{\partial \phi_{g}}{\partial r}=\frac{1}{\epsilon_{0}} \int_{0}^{\infty} d k\left[1-\frac{2}{k L} \tanh (k L / 2)\right] J_{1}(k r) \int_{0}^{\infty} d \tilde{r} \tilde{r} \rho_{b}(\tilde{r}) J_{0}(k \tilde{r}), \\
& -\int_{-L / 2}^{L / 2} \frac{d z}{L} \frac{\partial \phi_{g}}{\partial z}=0
\end{aligned}
$$

where $\tanh (x)=\sinh (x) / \cosh (x)$. For Gaussian- and uniform-density beams with $\rho_{b}(r)$ given by Eq. (6), we calculate

$$
\int_{0}^{\infty} d \tilde{r} \tilde{r} \rho_{b}(\tilde{r}) J_{0}(k \tilde{r})=\frac{\lambda}{\pi} \begin{cases}\frac{1}{2} e^{-k^{2} \sigma_{x}^{2} / 2}, & \text { Gaussian, } \\ \frac{1}{k r_{b}} J_{1}\left(k r_{b}\right), & \text { uniform, }\end{cases}
$$

and the average radial electric field in Eq. (27) reduces to

$$
-\int_{-L / 2}^{L / 2} \frac{d z}{L} \frac{\partial \phi_{g}}{\partial r}=\frac{\lambda}{\pi \epsilon_{0}} \begin{cases}\frac{1}{2} \int_{0}^{\infty} d k\left[1-\frac{2}{k L} \tanh (k L / 2)\right] e^{-k^{2} \sigma_{x}^{2} / 2} J_{1}(k r), & \text { Gaussian, } \\ \frac{1}{r_{b}} \int_{0}^{\infty} \frac{d k}{k}\left[1-\frac{2}{k L} \tanh (k L / 2)\right] J_{1}\left(k r_{b}\right) J_{1}(k r), & \text { uniform. }\end{cases}
$$

In Fig. 5, the $z$-averaged radial field in Eq. (29) is plotted as a function of $r$ for Gaussian- and uniform-density beams for families of beam radius to foil spacing aspect ratio (characteristic transverse beam extent to foil spacing). The aspect ratio is measured by $\sigma_{x} / L$ and $r_{b} / L$ for Gaussian- and uniform-density beams, respectively.
Small aspect-ratio limit results from Eq. (23) plotted agree well with curves for small aspect ratios. Large aspect-ratio results from Eq. (26) also agree well with the Gaussianbeam plots but are not shown since they would be compressed at the bottom range scale of the plots. For the uniform charge-density beam, the field is nearly linear 


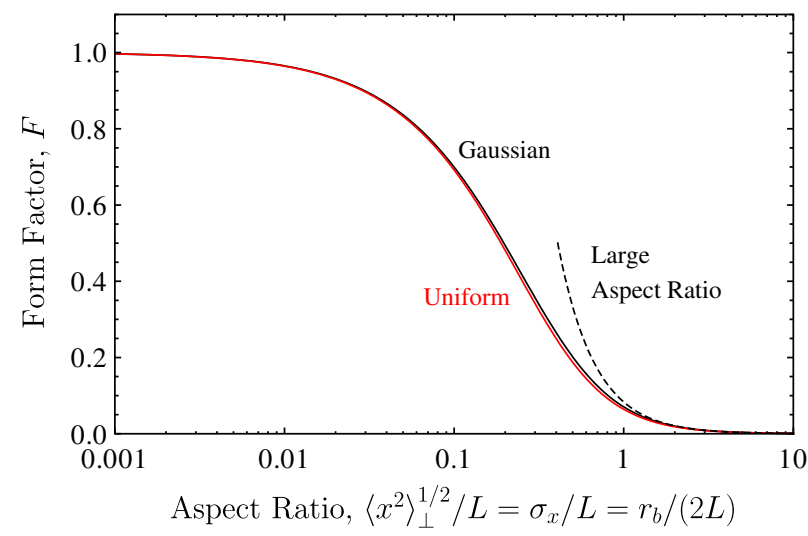

FIG. 6. Form factor $F$ [Eq. (49)] versus aspect ratio $\left\langle x^{2}\right\rangle_{\perp}^{1 / 2} / L=\sigma_{x} / L=r_{b} /(2 L)$ for Gaussian (black) and uniform (red) charge-density beams. rms-equivalent beams with $\sigma_{x}=$ $r_{b} / 2$ are plotted to allow direct comparison of the two cases. Limiting form results [Eq. (51)] valid for large aspect ratios with $\sigma_{x} / L=r_{b} /(2 L) \gg 1$ are plotted (black, dashed). The large aspect-ratio curve applies both to Gaussian and uniform beams.

within the beam for small aspect ratios $r_{b} / L$ and becomes progressively more nonlinear and peaked near the radial edge of the beam as $r_{b} / L$ becomes larger. Gaussiancharge-density results are qualitatively similar in scaling with aspect ratio to the uniform-density beam results, but the field is intrinsically nonlinear even for small aspect ratio $\sigma_{x} / L$ due to the Gaussian form of the beam charge density. Note that in both the Gaussian- and uniformdensity cases an aspect-ratio factor $\left(\sigma_{x} / L\right.$ and $\left.r_{b} / L\right)$ is included in the ordinate scale of the plot. This choice allows inclusion of small aspect-ratio limit results on a finite scale to better illustrate changes in radial field structure due to changes in relative foil spacing. Because of this scale factor, care should be taken not to directly interpret differences between curves in Fig. 5 as an attenuation factor. The curves also have different nonlinear structure for different aspect ratios. Therefore, differences between curves cannot be interpreted in terms of a simple attenuation factor. However, in Sec. IV B, a form factor $F \in(0,1)$ (see Fig. 6) is derived consistent with nonlinear effects which can be interpreted as an effective attenuation factor applied to the vacuum radial electric field to obtain the effect of the foils on decreasing the defocusing strength on the statistical beam evolution.

\section{TRANSVERSE ENVELOPE MODEL}

A transverse envelope model is derived for the statistical size of the beam. First, equations of motion are derived for the transverse particle trajectories (Sec. IVA). Then the trajectory equations are averaged over the distribution of particles within the beam (Sec. IV B) to obtain statistical envelope equations for the transverse size of the beam. Limits of the envelope equation are checked. Then the envelope equations are applied (Sec. IV C) to clarify under what conditions self-magnetic focusing can be stronger than self-electric defocusing for pinch focusing with minimal transverse spot size.

\section{A. Particle trajectory equations}

We examine the dynamics of a charged particle evolving between two foils in the presence of beam produced magnetic and electric fields and possibly a superimposed solenoidal magnetic field. Results can be applied anywhere in a long stack of foils by making appropriate axial translations. For present purposes, the beam self-electric field $\mathbf{E}$ and the self-magnetic field $\mathbf{B}$ are first only assumed to be consistent with an axisymmetric beam in the paraxial approximation, but we will later apply the field solutions derived in Sec. III. Linear focusing components of a superimposed solenoidal magnetic field $\mathbf{B}^{a}$ consistent with Eq. (1) is included. The particle is considered relatively energetic so we neglect changes in axial kinetic energy from penetrating the foils as well as any applied electric field resulting from the presence of any foil biases [i.e., $\left.\left|q\left(V_{l}-V_{r}\right)\right| / \mathcal{E}_{b} \ll 1\right]$, as well as the longitudinal component of the self-electric field produced by the beam between the foils. Neglecting scattering in the foils, the transverse particle trajectory equation can then be expressed within the paraxial approximation as [28]

$$
\begin{aligned}
\mathbf{x}_{\perp}^{\prime \prime} \simeq & \frac{q}{m \gamma_{b} \beta_{b} c} \hat{\mathbf{z}} \times \mathbf{B}_{\perp}^{a}+\frac{q B_{z}^{a}}{m \gamma_{b} \beta_{b} c} \mathbf{x}_{\perp}^{\prime} \times \hat{\mathbf{z}} \\
& +\left(1-\mathcal{F}_{J}\right) \times \frac{q}{m \gamma_{b} c^{2}} \frac{\partial \phi_{v}}{\partial \mathbf{x}_{\perp}} \\
& -\left(1-\mathcal{F}_{\rho}\right) \frac{q}{m \gamma_{b} \beta_{b}^{2} c^{2}} \frac{\partial \phi_{g}}{\partial \mathbf{x}_{\perp}} .
\end{aligned}
$$

Here and henceforth, primes denote derivatives with respect to $z$ (i.e., ${ }^{\prime}=\frac{d}{d z}$ ). Note in Eq. (30) that the coefficients of the self-field terms for magnetic focusing $\propto \frac{\partial \phi_{v}}{\partial \mathbf{x}_{\perp}}$ and for electric defocusing $\propto \frac{\partial \phi_{g}}{\partial \mathbf{x}_{\perp}}$ differ by a factor of $1 / \beta_{b}^{2}$.

If there is an applied solenoidal magnetic field [see Eq. (1)] with $B_{z 0} \neq 0$, the axisymmetric beam will rotate about the $z$ axis due to the cross-coupled form of the equation of motion (30). In this case Eq. (30) can be interpreted in a rotating Larmor frame (see Appendix B) to reduce to the uncoupled form

$$
\begin{gathered}
\mathbf{x}_{\perp}^{\prime \prime}+\kappa \mathbf{x}_{\perp}-\left(1-\mathcal{F}_{J}\right) \frac{q}{m \gamma_{b} c^{2}} \frac{\partial \phi_{v}}{\partial \mathbf{x}_{\perp}} \\
\simeq-\left(1-\mathcal{F}_{\rho}\right) \frac{q}{m \gamma_{b} \beta_{b}^{2} c^{2}} \frac{\partial \phi_{g}}{\partial \mathbf{x}_{\perp}} .
\end{gathered}
$$

Here,

$$
\kappa \equiv\left(\frac{B_{z 0}}{2[B \rho]}\right)^{2}=\left(\frac{q B_{z 0}}{2 m \gamma_{b} \beta_{b} c}\right)^{2}
$$


is the applied-focusing function where $[B \rho]=m \gamma_{b} \beta_{b} c / q$ denotes the particle rigidity. The Larmor-frame transformation does not influence the transverse size of the axisymmetric beam measured by $\left\langle x^{2}\right\rangle_{\perp}=\left\langle r^{2}\right\rangle_{\perp} / 2$. Note that Eq. (31) is trivially identical to Eq. (30) in laboratoryframe variables when $B_{z 0}=0$ (giving $\mathbf{B}^{a}=0$ and $\kappa=0$ ).

\section{B. Envelope equations}

To derive statistical envelope equations for the transverse size of the beam, we first take $z$ derivatives of $\left\langle x^{2}\right\rangle_{\perp}^{1 / 2}$ and apply the Larmor-frame equation of motion (31) to obtain

$$
\begin{aligned}
\frac{d}{d z}\left\langle x^{2}\right\rangle_{\perp}^{1 / 2} & =\frac{\left\langle x x^{\prime}\right\rangle_{\perp}}{\left\langle x^{2}\right\rangle_{\perp}^{1 / 2}} \\
\frac{d^{2}}{d z^{2}}\left\langle x^{2}\right\rangle_{\perp}^{1 / 2} & =\frac{\left\langle x x^{\prime \prime}\right\rangle_{\perp}}{\left\langle x^{2}\right\rangle_{\perp}^{1 / 2}}+\frac{\varepsilon_{x, \mathrm{rms}}^{2}}{\left\langle x^{2}\right\rangle_{\perp}^{3 / 2}}
\end{aligned}
$$

Here,

$$
\varepsilon_{x, \mathrm{rms}} \equiv\left[\left\langle x^{2}\right\rangle_{\perp}\left\langle x^{\prime 2}\right\rangle_{\perp}-\left\langle x x^{\prime}\right\rangle_{\perp}^{2}\right]^{1 / 2} \geq 0
$$

is the transverse rms emittance measured in the Larmor frame, which provides a statistical measure of the transverse phase-space area of the beam [28,32,36,37]. If there is no solenoidal magnetic field $\left(B_{z 0}=0\right)$, then the implicit Larmor-frame $x-x^{\prime}$ variables employed (see Appendix B) become the usual $x-x^{\prime}$ laboratory-frame variables and Eq. (34) is then the familiar expression for the rms emittance. The beam canonical angular momentum can be expressed in terms of laboratory-frame variables as

$$
P_{\theta} \equiv\left\langle x y^{\prime}-y x^{\prime}\right\rangle_{\perp}+\frac{q B_{z 0}}{2 m \gamma_{b} \beta_{b} c}\left\langle x^{2}+y^{2}\right\rangle_{\perp}=\mathrm{const}
$$

and is invariant (see Appendix B) for any axisymmetric beam evolution. For the case of no solenoidal magnetic field $\left(B_{z 0}=0\right)$ and a beam distribution with no imposed macroscopic rotation about the $z$ axis, $P_{\theta}=0$. It is shown in Appendix $B$ that if there is an axial magnetic field $\left(B_{z 0} \neq 0\right)$ and/or finite-beam canonical angular momentum $\left(P_{\theta} \neq 0\right)$, then Eq. (33) can be applied in laboratoryframe variables if the rms emittance (34) is replaced according to

$$
\varepsilon_{x, \mathrm{rms}}^{2} \rightarrow \varepsilon_{x, \mathrm{rms}}^{2}-\frac{1}{4}\left\langle r^{2} \theta^{\prime}\right\rangle_{\perp}^{2}+\frac{1}{4} P_{\theta}^{2}
$$

for proper laboratory-frame interpretation. The term related to $\left\langle r^{2} \theta^{\prime}\right\rangle_{\perp}^{2}$ on the right-hand side of Eq. (36) is associated with subtraction of the axial flow velocity (see Appendix B) so that a correct phase-space measure is obtained when $B_{z 0} \neq 0$ and/or $P_{\theta} \neq 0$.

We assume that $\varepsilon_{x, \text { rms }} \simeq$ const for the case of $B_{z 0}=0$ and $P_{\theta}=0$ and the substitution in Eq. (36) is likewise conserved when $B_{z 0} \neq 0$ and/or $P_{\theta} \neq 0$. These conservations of emittance measures can be shown to strictly hold only when transverse forces acting on the particles are linear $[28,38]$. In the present model, the space-charge force generally contains nonlinear terms. However, for short propagation distances in $z$, emittance evolutions can be limited. Moreover, in some systems the emittance term can be negligibly small relative to other terms in the moment equations rendering any modest evolution in emittance insignificant in determining the beam size outside of situations where the beam is focused to a spot with small $\left\langle x^{2}\right\rangle_{\perp}^{1 / 2}$. In more elaborate analysis, any significant evolution of $\varepsilon_{x, \text { rms }}$ can be modeled by more advanced theory [21] or simulations (see Sec. VI) and applied in the reduced model to improve accuracy. Formulas are given in Appendix $\mathrm{C}$ to estimate the beam emittance and canonical angular momentum in situations likely to be encountered in many applications.

We insert the $x$-component particle equation of motion from Eq. (31) in Eq. (33), and apply system axisymmetry to express $\left\langle x \frac{\partial \phi_{j}}{\partial x}\right\rangle_{\perp}=\frac{1}{2}\left\langle r \frac{\partial \phi_{j}}{\partial r}\right\rangle_{\perp}$ with $j=g$, $v$. This gives

$$
\begin{gathered}
\frac{d^{2}}{d z^{2}}\left\langle x^{2}\right\rangle_{\perp}^{1 / 2}+\kappa(z)\left\langle x^{2}\right\rangle_{\perp}^{1 / 2}-\left(1-\mathcal{F}_{J}\right) \frac{q}{2 m \gamma_{b} c^{2}} \frac{\left\langle r \frac{\partial \phi_{v}}{\partial r}\right\rangle_{\perp}}{\left\langle x^{2}\right\rangle_{\perp}^{1 / 2}} \\
+\left(1-\mathcal{F}_{\rho}\right) \frac{q}{2 m \gamma_{b} \beta_{b}^{2} c^{2}} \frac{\left\langle r \frac{\partial \phi_{g}}{\partial r}\right\rangle_{\perp}}{\left\langle x^{2}\right\rangle_{\perp}^{1 / 2}}-\frac{\varepsilon_{x, \mathrm{rms}}^{2}}{\left\langle x^{2}\right\rangle_{\perp}^{3 / 2}}=0
\end{gathered}
$$

First, we simplify the magnetic focusing term $\propto\left\langle r \frac{\partial \phi_{v}}{\partial r}\right\rangle_{\perp}$ in Eq. (37). For any axisymmetric beam charge distribution $\rho_{b}(r)$, it follows from the vacuum Poisson equation (9) that

$\left\langle r \frac{\partial \phi_{v}}{\partial r}\right\rangle_{\perp}=2\left\langle x \frac{\partial \phi_{v}}{\partial x}\right\rangle_{\perp}=-\frac{\lambda}{4 \pi \epsilon_{0}}=-\frac{I}{4 \pi \epsilon_{0} \beta_{b} c}$.

Here, $\lambda=q \int d^{2} x_{\perp} \int d^{3} p f_{b}=I /\left(\beta_{b} c\right)$ is the line charge of the beam at axial coordinate $z$. Equation (38) is a well-known result in the transverse physics of unbunched beams $[28,39]$.

Next, to simplify the electric defocusing term $\propto\left\langle r \frac{\partial \phi_{g}}{\partial r}\right\rangle_{\perp}$ in Eq. (37), we assume that the axisymmetric beam charge density $\rho_{b}(r)$ is known and apply the Green's function formulation results in Sec. III B for $-\frac{\partial \phi_{g}}{\partial r}$. In this procedure, we allow $\rho_{b}(r)$ to vary slowly in $z$ as the transverse size of the beam measured by $\left\langle x^{2}\right\rangle_{\perp}^{1 / 2}$ evolves. It is convenient to define a dimensionless "form factor"

$$
F(z) \equiv-\frac{4 \pi \epsilon_{0}}{\lambda}\left\langle r \frac{\partial \phi_{g}}{\partial r}\right\rangle_{\perp}
$$

to express the value of the moment. For closely spaced foils (i.e., $L$ small), the electric field $-\frac{\partial \phi_{g}}{\partial r}$ varies almost parabolically in $z$ from zero at the left and right foils at $z=$ $\pm L / 2$, to a maximum value between the foils at $z=0$. This results in a rapid $z$ variation of the electric defocusing force. Because a typical particle moves little radially while traversing between two closely spaced foils, we take a multiscale approximation and replace $F$ by its $z$-averaged value between the foils with 


$$
F \simeq-\frac{4 \pi \epsilon_{0}}{\lambda}\left\langle r \int_{-L / 2}^{L / 2} \frac{d z}{L} \frac{\partial \phi_{g}}{\partial r}\right\rangle_{\perp}
$$

Equation (40) constitutes a continuous defocusing approximation.

To reduce Eq. (37), we apply Eq. (38) in the magnetic focusing term and Eq. (39) [or alternatively, Eq. (40)] in the electric defocusing term and obtain the envelope equation

$$
\begin{aligned}
& \frac{d^{2}}{d z^{2}}\left\langle x^{2}\right\rangle_{\perp}^{1 / 2}+\kappa(z)\left\langle x^{2}\right\rangle_{\perp}^{1 / 2} \\
& \quad+\frac{\gamma_{b}^{2}}{4}\left[\left(1-\mathcal{F}_{J}\right) \beta_{b}^{2}-\left(1-\mathcal{F}_{\rho}\right) F\right] \frac{Q}{\left\langle x^{2}\right\rangle_{\perp}^{1 / 2}} \\
& \quad-\frac{\varepsilon_{x, \mathrm{rms}}^{2}}{\left\langle x^{2}\right\rangle_{\perp}^{3 / 2}}=0 .
\end{aligned}
$$

Here,

$$
Q \equiv \frac{q \lambda}{2 \pi \epsilon_{0} m \gamma_{b}^{3} \beta_{b}^{2} c^{2}}=\frac{q I}{2 \pi \epsilon_{0} m \gamma_{b}^{3} \beta_{b}^{3} c^{3}}=\text { const }
$$

is the usual dimensionless perveance from accelerator physics for the beam in the absence of neutralizing electrons $[28,36,37]$, and

$$
\begin{aligned}
F= & \frac{4 \pi}{\lambda} \int_{0}^{\infty} d k \frac{\cosh (k L / 2)-\cosh (k z)}{\cosh (k L / 2)}\left\langle r J_{1}(k r)\right\rangle_{\perp} \\
& \times \int_{0}^{\infty} d r r \rho_{b}(r) J_{0}(k r) \\
\simeq & \frac{4 \pi}{\lambda} \int_{0}^{\infty} d k\left[1-\frac{2}{k L} \tanh (k L / 2)\right]\left\langle r J_{1}(k r)\right\rangle_{\perp} \\
& \times \int_{0}^{\infty} d r r \rho_{b}(r) J_{0}(k r)
\end{aligned}
$$

is the simplified form factor obtained (first line, exact form) by using Eq. (21) for $-\frac{\partial \phi_{g}}{\partial r}$ including full $z$ variation, and (second line, in approximate form) using Eq. (27) for $-\int_{-L / 2}^{L / 2} \frac{d z}{L} \frac{\partial \phi_{g}}{\partial r}$ in the continuous defocusing approximation. The form factor $F$ is bounded by $0 \leq F \leq$ 1 and can be interpreted as an effective attenuation factor: the expression of the electric defocusing term in the absence of foils (i.e., in vacuum) should be multiplied by $F$ to account for the attenuation of the defocusing strength due to the foils. For the fixed functional form of the beam charge density $\rho_{b}(r)$ that we employ [e.g., Eq. (6)], $F$ can be expressed as a function of an aspectratio parameter formed from the parameter of dimension length used to set the transverse extent of $\rho_{b}(r)$ divided by $L$. Although the $z$-varying expression for $F$ is derived for a reference region between two particular foils, it can be applied anywhere in $z$ within the foil stack by appropriately shifting the $z$ location of $\phi_{g}$. Finite-thickness foils can be incorporated by inserting appropriate length zero-field regions where $\frac{-\partial \phi_{g}}{\partial r}=0$ within the foils. For the case of the continuous defocusing approximation, the expression for $F$ in the second line of Eq. (43) has no explicit dependence on $z$ and, provided the foil spacing $L$ remains constant, this expression can be applied throughout the lens.

Before explicitly calculating the form factor $F$ for Gaussian and uniform choices of $\rho_{b}(r)$ to fully specify the envelope model, it is instructive to interpret the terms in the envelope equation (41). First, $\kappa(z)\left\langle x^{2}\right\rangle_{\perp}^{1 / 2}$ represents the effect of linear applied-focusing forces from any solenoid magnetic field applied to the system with the focusing function $\kappa(z)$ specified by Eq. (32). The term $\frac{\left(\gamma_{b} \beta_{b}\right)^{2}}{4}\left(1-\mathcal{F}_{J}\right) \frac{Q}{\left\langle x^{2}\right\rangle_{\perp}^{1 / 2}}$ represents self-magnetic (z-pinch-type) focusing. The form of this term is independent of the specific form of $\rho_{b}(r)$ and becomes stronger as the transverse rms-beam width $\left\langle x^{2}\right\rangle_{\perp}^{1 / 2}$ becomes smaller and therefore constitutes a "pinching force" which within the paraxial approximation would focus the beam to a vanishingly small spot in the absence of other defocusing forces. However, the electric defocusing term $-\frac{\gamma_{b}^{2}}{4}\left(1-\mathcal{F}_{\rho}\right) \frac{Q F}{\left\langle x^{2}\right\rangle_{\perp}^{1 / 2}}$ and the defocusing emittance term $-\frac{\varepsilon_{x, \text { rms }}^{2}}{\left\langle x^{2}\right\rangle_{\perp}^{3 / 2}}$ (see following analysis) both become larger than the magnetic focusing term and ultimately limit the reduction of transverse rms-beam extent as $\left\langle x^{2}\right\rangle_{\perp}^{1 / 2}$ becomes sufficiently small. The relative strength of the magnetic focusing term is larger than the electric defocusing term when

$$
\beta_{b}>\sqrt{\frac{1-\mathcal{F}_{\rho}}{1-\mathcal{F}_{J}} F} .
$$

Further clarification on the scaling of $F$ and the electric defocusing term for explicit choices of beam charge density $\rho_{b}(r)$ is made below. Even when Eq. (44) is initially satisfied, the defocusing emittance term can limit the size of the pinch focused spot in $\left\langle x^{2}\right\rangle_{\perp}^{1 / 2}$ for any finite $\varepsilon_{x, \text { rms }}>0$, because the $1 /\left\langle x^{2}\right\rangle_{\perp}^{3 / 2}$ variation of the emittance term will eventually dominate the $1 /\left\langle x^{2}\right\rangle_{\perp}^{1 / 2}$ variation of the magnetic focusing term as the rms-beam width $\left\langle x^{2}\right\rangle_{\perp}^{1 / 2}$ decreases.

For $\rho_{b}$ corresponding to the case of a Gaussian-chargedensity beam in Eq. (6), $\left\langle x^{2}\right\rangle_{\perp}^{1 / 2}=\sigma_{x}$ and Eq. (41) can be denoted as

$$
\begin{aligned}
& \frac{d^{2}}{d z^{2}} \sigma_{x}+\kappa(z) \sigma_{x} \\
& \quad+\frac{\gamma_{b}^{2}}{4}\left[\left(1-\mathcal{F}_{J}\right) \beta_{b}^{2}-\left(1-\mathcal{F}_{\rho}\right) F\right] \frac{Q}{\sigma_{x}}-\frac{\varepsilon_{x, \mathrm{rms}}^{2}}{\sigma_{x}^{3}}=0 .
\end{aligned}
$$

For $\rho_{b}$ corresponding to the case of a uniform-chargedensity beam in Eq. (6), $\left\langle x^{2}\right\rangle_{\perp}^{1 / 2}=r_{b} / 2$ and the rmsenvelope equation can be equivalently expressed as 


$$
\begin{aligned}
& \frac{d^{2}}{d z^{2}} r_{b}+\kappa(z) r_{b} \\
& \quad+\gamma_{b}^{2}\left[\left(1-\mathcal{F}_{J}\right) \beta_{b}^{2}-\left(1-\mathcal{F}_{\rho}\right) F\right] \frac{Q}{r_{b}}-\frac{\varepsilon_{x}^{2}}{r_{b}^{3}}=0,
\end{aligned}
$$

where

$$
\varepsilon_{x} \equiv 4 \varepsilon_{x, \mathrm{rms}}
$$

denotes the usual "rms-edge emittance." To finish the envelope model descriptions for Gaussian- and uniformcharge-density beams, the form factor $F$ should be calculated. Using Eq. (28) and the moment

$$
\left\langle r J_{1}(k r)\right\rangle_{\perp}= \begin{cases}k \sigma_{x}^{2} e^{-k^{2} \sigma_{x}^{2} / 2}, & \text { Gaussian, } \\ \frac{2}{k} J_{2}\left(k r_{b}\right), & \text { uniform, }\end{cases}
$$

the continuous defocusing expression for $F$ in Eq. (43) can be reduced to a single integral with

$$
F= \begin{cases}2\left(\sigma_{x} / L\right)^{2} \int_{0}^{\infty} d K K\left[1-\frac{2}{K} \tanh (K / 2)\right] e^{-K^{2}\left(\sigma_{x} / L\right)^{2}}, & \text { Gaussian, } \\ \frac{8}{\left(r_{b} / L\right)} \int_{0}^{\infty} \frac{d K}{K^{2}}\left[1-\frac{2}{K} \tanh (K / 2)\right] J_{1}\left(K \frac{r_{b}}{L}\right) J_{2}\left(K \frac{r_{b}}{L}\right), & \text { uniform. }\end{cases}
$$

Here, we employ $K \equiv k L$ as a dimensionless integration variable to better illustrate the dependence of $F$ on the aspect-ratio parameters $\sigma_{x} / L$ and $r_{b} / L$. Similar steps can be applied to obtain reduced Gaussian and uniform-beam expressions for $F$ including full $z$ variation. For specified $\beta_{b}, L, \kappa(s), Q$, and $\varepsilon_{x, \text { rms }}$, Eqs. (45) and (49) can be applied to numerically solve for the evolution of $\sigma_{x}(z)$ for a Gaussian-charge-density beam from specified initial $\left(z=z_{i}\right)$ values $\sigma_{x}\left(z_{i}\right)=\sigma_{x i}$ and $\frac{d \sigma_{x}}{d z}\left(z_{i}\right)=\sigma_{x i}^{\prime}$ to model the evolution of the transverse beam size. To efficiently implement this numerical procedure, the Gaussian form factor $F$ in Eq. (49) can be precalculated as a function of $\sigma_{x} / L$ on a grid over an adequate range and then $F$ can be efficiently calculated to sufficient accuracy using interpolation to carry out the integrations without many evaluations of the form factor integral over $K$. Analogous comments apply to application to Eqs. (46) and (49) to model the evolution of $r_{b}$ for a uniform-density beam from specified initial conditions $r_{b}\left(z_{i}\right)=r_{b i}$ and $\frac{d r_{b}}{d z}\left(z_{i}\right)=r_{b i}^{\prime}$. Note that if $\sigma_{x} \rightarrow r_{b} / 2$ is substituted in Eq. (45) and $\varepsilon_{x}^{2}=16 \varepsilon_{x, \mathrm{rms}}^{2}$ is applied, then the uniform-beam envelope equation (46) is obtained. Thus, for rms-equivalent beams with $\left\langle x^{2}\right\rangle_{\perp}^{1 / 2}=\sigma_{x}=r_{b} / 2$, all differences between the Gaussian- and uniform-beam envelope equations are contained in the form factor $F$.

The form factor $F$ [Eq. (49)] in the continuous defocusing approximation is plotted in Fig. 6 as a function of the beam aspect ratio for Gaussian- and uniform-density beams. The curves are shown superimposed for rmsequivalent beam parameters with $\sigma_{x}=r_{b} / 2$. Note that there is comparatively little difference between the two curves. The maximum deviation of $F$ between the Gaussian- and uniform-rms-equivalent beams is 0.0157 at $\sigma_{x}=r_{b} / 2=0.345$. Differences in rms-equivalent Gaussian- and uniform-beam values of $F$ are very small for both small and large aspect ratios. Because all differences between rms-equivalent beams are contained in the form factor $F$, only relatively small differences are expected between the Gaussian- and uniform-beam evolution models if they are applied with proper rms equivalency on the initial conditions and parameters. It is reasonable to expect that a wide variety of other rms-equivalent chargedensity functions $\rho_{b}(r)$ which are monotonic in $r$ [i.e., $\frac{d \rho_{b}(r)}{d r} \leq 0$ ] and reasonably similar in radial profile should produce relatively close values of form factor $F$. In this regard, it is reasonable to expect the envelope evolution to be relatively insensitive to the details of the distribution when the emittance $\varepsilon_{x, \text { rms }}$ is nearly constant or negligibly small.

It is further instructive to examine limits of the form factor $F$. First, for small aspect ratios with $\sigma_{x} / L \ll 1$ or $r_{b} / L \ll 1$ it can be shown using arguments analogous to those employed in Eq. (38) that $F \rightarrow 1$ regardless of the beam density profile. In this limit, there is no difference between the rms-equivalent Gaussian- and uniform-beam cases and for an unneutralized beam with $\mathcal{F}_{\rho}=\mathcal{F}_{J}=0$, the magnetic and electric terms combine with $\gamma_{b}^{2}\left(\beta_{b}^{2}-F\right)=-1$ and the uniform-beam envelope equation (46) reduces to

$$
\frac{d^{2}}{d z^{2}} r_{b}+\kappa(z) r_{b}-\frac{Q}{r_{b}}-\frac{\varepsilon_{x}^{2}}{r_{b}^{3}}=0 .
$$

Equation (50) can be recognized as the familiar envelope equation describing an unbunched, uniform-density beam focused in an applied solenoidal magnetic field including space-charge and emittance defocusing forces [28,36,37]. This reduction to the free-space form in Eq. (50) can be shown to be independent of the transverse beam charge density for reasons analogous to those discussed in obtaining Eq. (38).

In the opposite limit of a large aspect-ratio beam with $\sigma_{x} / L \gg 1$ or $r_{b} / L \gg 1$, the limiting form of $-\int_{-L / 2}^{L / 2} \frac{d z}{L} \frac{\partial \phi_{g}}{\partial r}$ in Eq. (26) can be applied to show that the form factor expression in Eq. (43) in the approximation of continuous defocusing reduces to

$$
F \simeq \frac{L^{2}}{3} \begin{cases}\frac{1}{4 \sigma_{x}^{2}}, & \text { Gaussian, } \\ \frac{1}{r_{b}^{2}}, & \text { uniform }\end{cases}
$$


for the Gaussian- and uniform-charge-density cases. Equation (51) is formally valid for $\sigma_{x} / L \gg 1$ and $r_{b} / L \gg$ 1 in the Gaussian and uniform cases. Numerical tests show agreement to within 5\% fractional difference for $\sigma_{x} / L>$ 1.97 and $r_{b} / L>9.96$ for the Gaussian and uniform cases, but results break down rapidly for smaller aspect ratios (see Fig. 6). For beams with rms-equivalent aspect ratios with $\left\langle x^{2}\right\rangle_{\perp}^{1 / 2}=\sigma_{x}=r_{b} / 2$, note that the large aspect-ratio limit of $F$ given by Eq. (51) is exactly equal for Gaussian and uniform-density beams with $F=L^{2} /\left(12\left\langle x^{2}\right\rangle_{\perp}\right)$. From this result, the envelope equation (41) for either Gaussian- or uniform-density beams reduces for $\left\langle x^{2}\right\rangle_{\perp}^{1 / 2} / L \gg 1$ to

$$
\begin{gathered}
\frac{d^{2}}{d z^{2}}\left\langle x^{2}\right\rangle_{\perp}^{1 / 2}+\kappa(z)\left\langle x^{2}\right\rangle_{\perp}^{1 / 2}+\frac{\gamma_{b}^{2} \beta_{b}^{2}}{4}\left(1-\mathcal{F}_{J}\right) \frac{Q}{\left\langle x^{2}\right\rangle_{\perp}^{1 / 2}} \\
-\frac{\left(\gamma_{b}^{2} / 48\right)\left(1-\mathcal{F}_{\rho}\right) L^{2} Q+\varepsilon_{x, \mathrm{rms}}^{2}}{\left\langle x^{2}\right\rangle_{\perp}^{3 / 2}}=0 .
\end{gathered}
$$

This result explicitly shows that insofar as the aspect-ratio limit is maintained, the electric defocusing term with $\sim 1 /\left\langle x^{2}\right\rangle_{\perp}^{3 / 2}$ variation becomes stronger than the magnetic focusing term with $\sim 1 /\left\langle x^{2}\right\rangle_{\perp}^{1 / 2}$ variation for sufficiently small rms extent $\left\langle x^{2}\right\rangle_{\perp}^{1 / 2}$. Note that the electric defocusing term in Eq. (52) has the same $\sim 1 /\left\langle x^{2}\right\rangle_{\perp}^{3 / 2}$ variation as the defocusing emittance term so the electric defocusing appears analogous to an emittance term for large aspectratio beams.

\section{Requirements for magnetic pinch focusing}

A necessary condition to achieve magnetic pinch focusing with a possibly dramatically smaller transverse beam spot is that the magnetic focusing force must be larger than the electric defocusing force. The condition for this requirement is given by Eq. (44). Beam energies sufficiently high to satisfy this criterion can be focused to a smaller transverse spot size even in the absence of externally applied-focusing forces $(\kappa=0)$. The strength of the focusing is dictated by the values of the beam perveance $Q$ and any neutralization factors $\mathcal{F}_{\rho} \neq 0$ and $\mathcal{F}_{J} \neq 0$. Larger $Q$ for a given beam kinetic energy produces stronger focusing, which requires less propagation distance in $z$ and less foils to focus to a reduced spot. However, whether there is net focusing or not is independent of $Q$. In the continuous defocusing approximation, the form factor $F$ occurring in the pinch focusing condition (44) depends only on the beam aspect ratio, i.e., the transverse beam size relative to the foil separation. In Fig. 7(a), Eq. (49) is applied for the form factor in Eq. (44) to plot the minimum value of $\beta_{b}$ for which there is net focusing for an unneutralized beam $\left(\mathcal{F}_{\rho}=\mathcal{F}_{J}=0\right)$ as a function of beam aspect ratio for rmsequivalent Gaussian- and uniform-charge-density beams (i.e., $\sigma_{x}=r_{b} / 2$ ). The results in Fig. 7(a) are plotted for protons in Fig. 7(b) in terms of the minimum proton kinetic
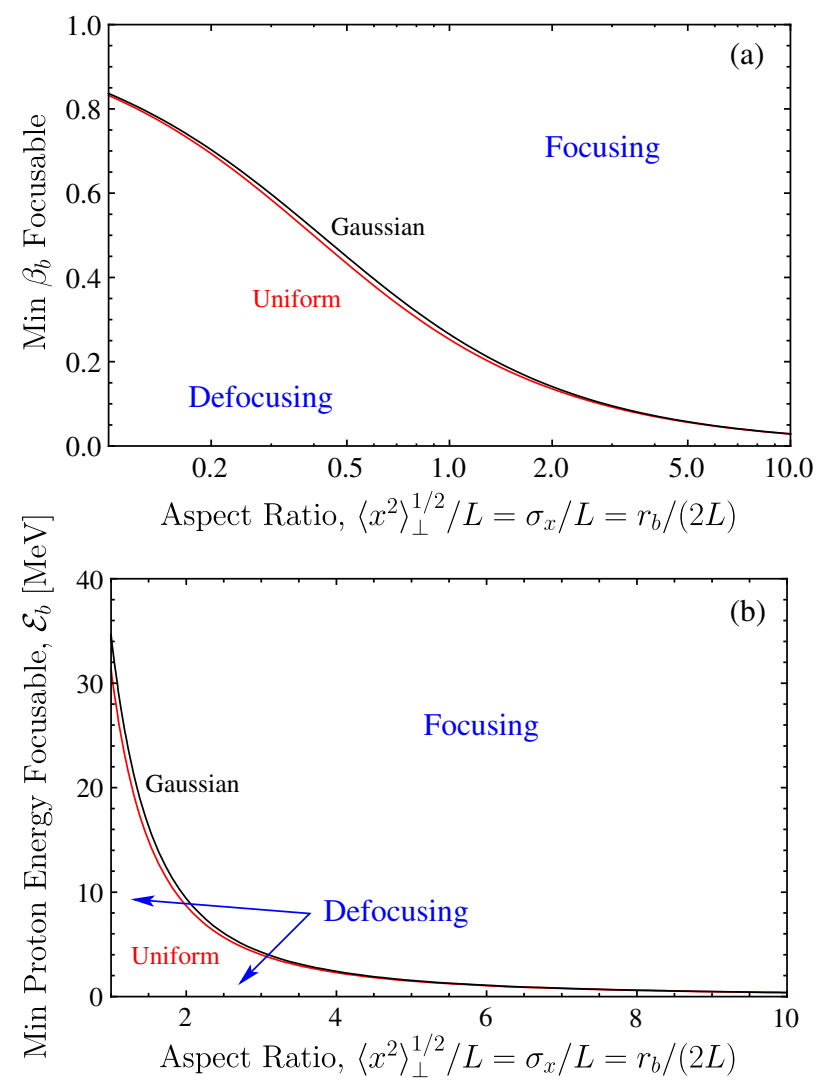

FIG. 7. (a) Minimum value of beam beta $\left(\beta_{b}\right)$ for the magnetic focusing term to be larger than the electric defocusing term plotted as a function of beam aspect ratio $\left\langle x^{2}\right\rangle_{\perp}^{1 / 2} / L=\sigma_{x} / L=$ $r_{b} /(2 L)$ for Gaussian (black) and uniform (red) charge-density beams. rms-equivalent beams with $\left\langle x^{2}\right\rangle_{\perp}^{1 / 2}=\sigma_{x}=r_{b} / 2$ are plotted to allow direct comparison of the two cases and no neutralization is assumed $\left(\mathcal{F}_{\rho}=\mathcal{F}_{J}=0\right)$. (b) Expanded scale results for protons expressed in terms of the minimum kinetic energy $\mathcal{E}_{b}=\left(\gamma_{b}-1\right) m c^{2}$.

energy $\mathcal{E}_{b}=\left(\gamma_{b}-1\right) m c^{2}$ for focusing. Note that there is little difference between the curves for the Gaussian and uniform cases. For beams with electron neutralization, charge neutralization $\left(\mathcal{F}_{\rho}>0\right)$ reduces the critical value of $\beta_{b}$, whereas current neutralization $\left(\mathcal{F}_{J}>0\right)$ increases the critical $\beta_{b}$. Beams with initial aspect ratios satisfying the bound will be focused. If the focusing coefficient is strong ( $Q$ large), the reduction in transverse beam size occurs rapidly within the lens until the aspect ratio becomes sufficiently small that the electric defocusing force overwhelms the magnetic focusing (see examples in Sec. V).

For the case of negligible electric defocusing corresponding to closely spaced foils $(L \rightarrow 0$ and $F \rightarrow 0$ ), no external solenoidal focusing $(\kappa=0)$, and negligible beam emittance $\left[\varepsilon_{x, \text { rms }} \rightarrow 0\right.$; this limit implicitly includes any system $P_{\theta}$ via the replacement in Eq. (36)], the rmsenvelope equation (41) reduces to 


$$
\frac{d^{2}}{d z^{2}}\left\langle x^{2}\right\rangle_{\perp}^{1 / 2}+\frac{Q_{\mathrm{eff}}}{\left\langle x^{2}\right\rangle_{\perp}^{1 / 2}}=0
$$

where

$$
Q_{\text {eff }} \equiv \frac{\left(\gamma_{b} \beta_{b}\right)^{2}}{4}\left(1-\mathcal{F}_{J}\right) Q
$$

denotes an effective perveance. In this idealized situation modeled within the paraxial approximation (i.e., changes in $\beta_{b}$ due to possibly large focusing angles neglected) with no electric defocusing force or emittance, the magnetic focusing force will ultimately "pinch" the beam to a zero rms-extent focal spot (i.e., $\left\langle x^{2}\right\rangle_{\perp}^{1 / 2} \rightarrow 0$; see example solutions in Sec. V). Such a limiting pinch cannot be achieved in the laboratory for a number of reasons. First, the limit $L=0$ corresponds to the beam propagating through solid metal and mobile electrons in the metal may neutralize the beam current reducing the magnetic focusing force leading to reduced $Q_{\text {eff }}$. Moreover, finitebeam emittance, nonparaxial particle dynamics, and other effects may also limit achievable spot size. Nevertheless, the idealization of taking $L=0$ within the context of a model appropriate for finite $L$ to remove the defocusing electric term while retaining the magnetic focusing term serves to clarify limits to obtainable decreases in the propagation distance to the spot as the foil spacing $L$ becomes smaller. Equation (53) can be integrated using the conserved envelope Hamiltonian (see Appendix D) to show that an initial $\left(z=z_{i}\right)$ beam with $\sigma_{x i}=\left.\left\langle x^{2}\right\rangle_{\perp}^{1 / 2}\right|_{z=z_{i}}$ and $\sigma_{x i}^{\prime}=\left.\frac{d}{d z}\left\langle x^{2}\right\rangle_{\perp}^{1 / 2}\right|_{z=z_{i}}$ will reach a pinched focal spot with $\left\langle x^{2}\right\rangle_{\perp}^{1 / 2}=0$ at $z=z_{f}$ with

$z_{f}-z_{i}=\sqrt{\frac{\pi}{2 Q_{\text {eff }}}} \exp \left(\frac{\sigma_{x i}^{\prime 2}}{2 Q_{\text {eff }}}\right) \sigma_{x i}\left[1+\operatorname{sgn}\left(\sigma_{x i}^{\prime}\right) \operatorname{erf}\left(\sqrt{\frac{\sigma_{x i}^{\prime 2}}{2 Q_{\text {eff }}}}\right)\right]$.

Here, $\operatorname{erf}(z) \equiv \frac{2}{\sqrt{\pi}} \int_{0}^{z} d t e^{-t^{2}}$ denotes the error function [35], and $\operatorname{sgn}(x)$ is a sign function defined by $\operatorname{sgn}(x)=1$ for $x \geq 0$ and $\operatorname{sgn}(x)=-1$ for $x<0$. Note that Eq. (55) applies to any radial charge distribution (not necessarily Gaussian) and we employ $\sigma_{x}=\left\langle x^{2}\right\rangle_{\perp}^{1 / 2}$ for notational convenience. For the special case of an initial beam with $\sigma_{x i}^{\prime}=0$, Eq. (55) reduces to

$$
z_{f}-z_{i}=\frac{1}{\gamma_{b} \beta_{b}} \sqrt{\frac{2 \pi}{\left(1-\mathcal{F}_{J}\right) Q}} \sigma_{x i} .
$$

These results show that higher perveance $Q$ results in more rapid focusing and provide a simple lower bound estimate of the characteristic propagation distance to focus in more realistic situations (see examples in Sec. V).

The envelope Hamiltonian can provide a useful constraint to estimate the achievable focusing spot in a foil lens in less idealized situations where a full pinch is not achieved. Denoting $\sigma_{x}=\left\langle x^{2}\right\rangle_{\perp}^{1 / 2}$ for any charge distribution (not necessarily Gaussian) for notational convenience, the Hamiltonian of the rms-envelope equation (41) is

$$
H=\frac{1}{2} \sigma_{x}^{\prime 2}+\frac{1}{2} \kappa \sigma_{x}^{2}+Q_{\mathrm{eff}} \ln \sigma_{x}+V+\frac{\varepsilon_{x, \mathrm{rms}}^{2}}{2 \sigma_{x}^{2}} .
$$

Here, $V$ is a potential associated with electric defocusing forces which satisfies

$$
-\frac{\partial V}{\partial \sigma_{x}}=\frac{\gamma_{b}^{2}}{4}\left(1-\mathcal{F}_{\rho}\right) Q \frac{F}{\sigma_{x}} .
$$

If $\kappa=$ const (including zero corresponding to no solenoid focusing) and the approximate $z$-independent form of the form factor $F$ is applied corresponding to continuous defocusing [the second form of $F$ given in Eq. (43)], then there is no explicit $z$ dependence and $H=$ const. When $H=$ const, the expression for $H$ in Eq. (57) can be equated between the initial beam with $\sigma_{x}=\sigma_{x i}$ and $\sigma_{x}^{\prime}=\sigma_{x i}^{\prime}$ at $z=z_{i}$ and the beam at the focal spot where $\sigma_{x}=\sigma_{x f}$ and $\sigma_{x}^{\prime}=0$ at $z=z_{f}$ to give an expression which can be numerically solved for the rms-beam width at the focal spot $\left(\sigma_{x f}\right)$. For a Gaussian-charge-density beam with $F$ given by Eq. (49) Eq. (58) can be solved for $V$ as

$$
\begin{aligned}
V= & \frac{\gamma_{b}^{2}}{4}\left(1-\mathcal{F}_{\rho}\right) Q \int_{0}^{\infty} \frac{d K}{K}\left[1-\frac{2}{K} \tanh (K / 2)\right] \\
& \times e^{-K^{2}\left(\sigma_{x} / L\right)^{2}}+\text { const. }
\end{aligned}
$$

An analytical solution for $V$ for a uniform-charge-density beam can also be derived in terms of an equation based on a hypergeometric function but is not particularly insightful. For the special case of a full pinch focus which occurs with $L \rightarrow 0$ and no electric focusing (giving $V=$ const) and $\varepsilon_{x, \text { rms }}=0$, the Hamiltonian constraint can be applied with $\sigma_{x f} \rightarrow 0$ to solve for the final pinch angle $\sigma_{x f}^{\prime}<0$. To illustrate the method under less idealized situations, we examine the case of a Gaussian beam satisfying the large aspect-ratio limit $\sigma_{x} / L \gg 1$. Then the reduced form factor expression in Eq. (51) can be applied to solve Eq. (58) as $V=\frac{\gamma_{b}^{2}}{96}\left(1-\mathcal{F}_{\rho}\right) Q \frac{L^{2}}{\sigma_{x}^{2}}+$ const. Using this result in Eq. (57) for the Hamiltonian and equating the initial and final value of the Hamiltonian then gives the constraint

$$
\begin{gathered}
\frac{1}{2} \sigma_{x i}^{\prime 2}+\frac{1}{2} \kappa\left(\sigma_{x i}^{2}-\sigma_{x f}^{2}\right)+Q_{\mathrm{eff}} \ln \left(\frac{\sigma_{x i}}{\sigma_{x f}}\right) \\
+\frac{\varepsilon_{x, \mathrm{eff}}^{2}}{2}\left(\frac{1}{\sigma_{x i}^{2}}-\frac{1}{\sigma_{x f}^{2}}\right)=0 .
\end{gathered}
$$

Here,

$$
\varepsilon_{x, \mathrm{eff}}^{2} \equiv \varepsilon_{x, \mathrm{rms}}^{2}+\frac{\gamma_{b}^{2}}{48}\left(1-\mathcal{F}_{\rho}\right) L^{2} Q
$$

is an effective emittance which includes the effects of the electric defocusing force for a large aspect-ratio Gaussian 
beam. Note that $\varepsilon_{x, \text { rms }}$ can also include finite system canonical angular momentum through the replacement in Eq. (36). Equation (60) is a transcendental equation which can be numerically solved for $\sigma_{x f}$ to rapidly explore the achievable spot size for specified system and initial beam parameters. Results obtained will be accurate insofar as both $\sigma_{x i} / L$ and $\sigma_{x f} / L$ are sufficiently large where Eq. (51) remains a good approximation.

\section{EXAMPLE: APPLICATION OF THE ENVELOPE MODEL TO AN IDEALIZED PROTON BEAM}

Because little difference is found between the envelope models for rms-equivalent beams with Gaussian- and uniform-charge density derived in Sec. IV, we present an illustrative example for the Gaussian case in this section before analyzing ranges of variation possible if the distribution is different at the end of the section. For simplicity, we apply the continuous defocusing form of the form factor $F$ in Eq. (49) and thereby neglect the rapid $z$ variation of $F$ between foils. The large aspect limiting form of $F$ in Eq. (51) is not applied to avoid needing to consider ranges where validity of the simpler limiting expression breaks down in cases where the beam is strongly focused. Because the focusing action of linear applied fields is well understood in envelope models $[28,36]$, we consider envelope evolution in the absence of additional applied focusing $(\kappa=0)$ which can be provided by a superimposed solenoidal field. However, comments will be made on situations where such additional focusing could be beneficial. We only present envelope solutions with zero emittance $\left(\varepsilon_{x, \text { rms }}=0\right)$ and zero canonical angular momentum $\left(P_{\theta}=0\right)$. This is assumed because our example is partially motivated by laser-produced proton sources, which typically generates a nearly cold beam, implying negligibly small emittance. Such beams are also usually produced outside of an applied magnetic field, which implies zero canonical angular momentum (i.e., $P_{\theta}=0$, see Appendix C). Moreover, neglecting emittance and angular momentum more simply emphasizes effects due to the relative balance of focusing magnetic and defocusing electric self-field forces acting on the beam-which is the primary subject of this study. For simplicity, we consider a fully unneutralized beam with $\mathcal{F}_{\rho}=\mathcal{F}_{J}=0$. It is straightforward to modify analysis to evaluate the implications of partial neutralization with $\mathcal{F}_{\rho} \neq 0$ and $\mathcal{F}_{J} \neq 0$.

We consider parameters idealized from a recently performed foil focusing experiment using a TNSA produced proton beam at the PHELIX laser facility at GSI [10]. The parameters employed here can be considered modest and idealized and serve to illustrate basic focusing properties. Results from the experiment will be reported separately. It should also be stressed that parameters associated with laser-produced proton beams will likely evolve as technology and methods develop. Higher proton-beam energies and currents are available on a variety of other short pulse laser facilities [2,5], so experiments to verify passive focusing can be carried out on facilities with a more favorable range of parameters. For present purposes, a $\mathcal{E}_{b}=10 \mathrm{MeV}$ portion of the beam with $\sim 10^{10}$ protons in a 4 ps window of the pulse is analyzed as a long pulse with negligible energy spread. These parameters correspond to a beam current of $I \simeq 400.5$ A (chosen to be approximately that in a $1 \mathrm{MeV}$ window about $10 \mathrm{MeV}$ in the full PHELIX-produced beam) and dimensionless perveance $Q=8.13 \times 10^{-3}$. This is a strong idealization of typical TNSA proton beams which have a broad, roughly exponentially varying energy spectrum. However, methods exist to produce a more monochromatic energy distribution by restricting the source region within the TNSA context to a small volume [40-42], or alternatively, through exploiting a radiation-pressureinduced acceleration mechanism [43,44]. The initial $\left(z=z_{i}=0\right)$ rms-beam width $\sigma_{x}\left(z=z_{i}\right)=\sigma_{x i}$ and divergence $\left.\frac{d \sigma_{x}}{d z}\right|_{z=z_{i}}=\sigma_{x i}^{\prime}$ of the Gaussian beam is assumed to be manipulated by shaping the proton emitting side of the foil illuminated by the laser and adjusting the axial standoff distance of the emitting surface to the thin-foil lens $[5,45,46]$. Initial rms-beam widths with $\sigma_{x i} \sim 200 \mu \mathrm{m}$ and envelope divergence angles $\sigma_{x i}^{\prime}$ with $\sigma_{x i}^{\prime} \sim 393 \mathrm{mrad}$ $\left(22.5^{\circ}\right)$ have been demonstrated with no shaping of the emitting foil and relatively long $(\sim 20 \mathrm{~mm})$ stand-off distance. It is believed possible to achieve similar scale $\sigma_{x i}$ with $\sigma_{x i}^{\prime} \sim 0$ or even converging with $\sigma_{x i}^{\prime}<0$ if the emitting foil and the stand-off distance are appropriately tuned [5]. PIC simulations are employed in Sec. VI to address changes in the idealized results in this section resulting from somewhat more realistic conditions. Issues on distribution evolution and halo generation, short pulse length, and large energy spread combined with short pulse length are examined. It is found that basic focusing properties illustrated here largely persist under less idealized conditions.

First, we calculate the required beam aspect ratio for net focusing with $\mathcal{E}_{b}=10 \mathrm{MeV}$ using Eq. (44) [see also Fig. 7(b)] as $\sigma_{x} / L=1.94$. For a beam with an initial rms width $\sigma_{x i}=200 \mu \mathrm{m}$, this requires a foil spacing of $L<$ $103 \mu \mathrm{m}$ for focusing. In Fig. 8, envelope evolution of $\sigma_{x}$ versus $z$ obtained from numerical solutions to Eqs. (45) and (49) are plotted for round-number increments of foil spacing $L$ about the critical value of $L=103 \mu \mathrm{m}$. Zero initial envelope divergence is assumed $\left(\sigma_{x i}^{\prime}=0\right)$. For small foil spacing with $L<100 \mu \mathrm{m}$, the beam focuses with decreasing $\sigma_{x}$ until the aspect ratio $\sigma_{x} / L$ increases to where the electric defocusing force becomes larger than the magnetic focusing force and causes $\sigma_{x}$ to "bounce" up to larger values. Then favorable aspect ratio is achieved again and the focusing cycle repeats. Note that at the minimum value of $\sigma_{x}$, the aspect ratio $\sigma_{x} / L$ is significantly smaller (see data in Table I) than the critical value $\left(\sigma_{x} / L=1.94\right)$ where the electric force becomes stronger than the magnetic force. This can be understood as follows. When the aspect ratio $\sigma_{x i} / L$ of the initial beam is consistent with 

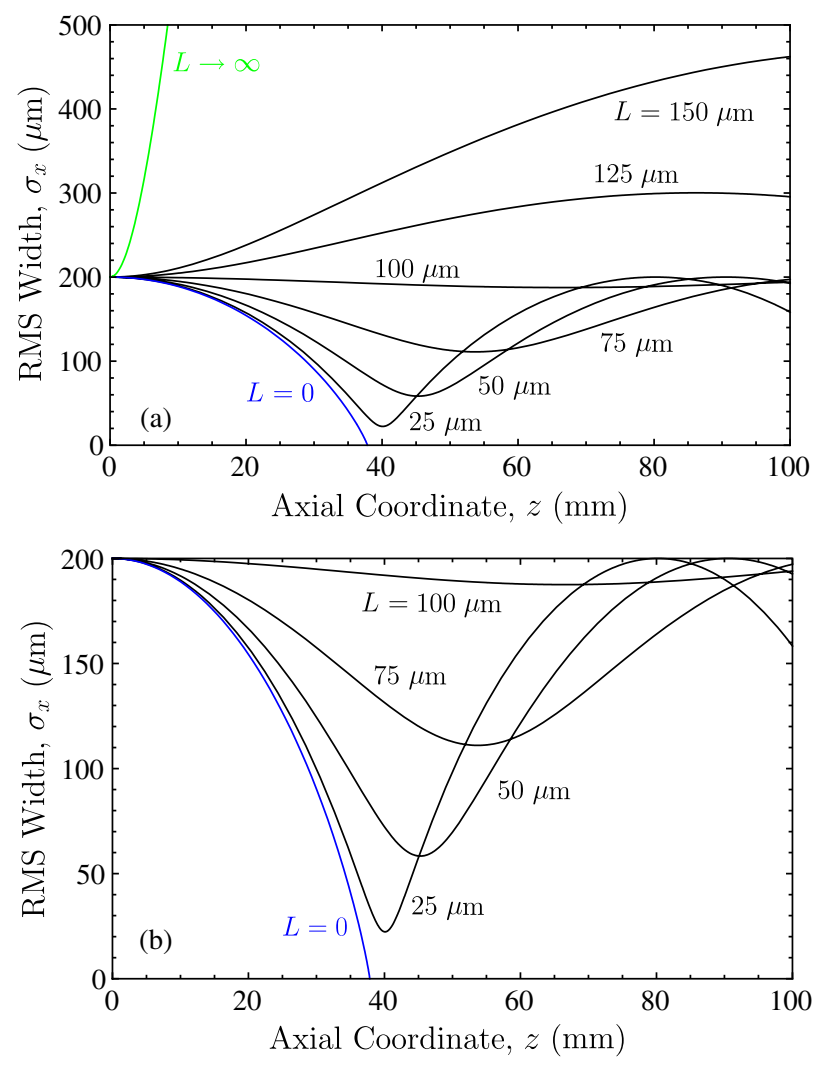

FIG. 8. (a) Envelope evolution for the transverse rms-beam width $\sigma_{x}=\left\langle x^{2}\right\rangle_{\perp}^{1 / 2}$ versus $z$ for a Gaussian-density proton beam with $\mathcal{E}_{b}=10 \mathrm{MeV}$ and $I=400.5 \mathrm{~A}$. Initial $\left(z=z_{i}\right)$ conditions are $\sigma_{x}=\sigma_{x i}=200 \mu \mathrm{m}, \sigma_{x}^{\prime}=\sigma_{x i}^{\prime}=0$ and solutions are shown for a range of foil spacings $L$ as indicated. The beam has zero emittance $\left(\varepsilon_{x, \mathrm{rms}}=0\right)$ and canonical angular momentum $\left(P_{\theta}=0\right)$, no applied magnetic focusing $(\kappa=0)$, and no neutralization $\left(\mathcal{F}_{\rho}=\mathcal{F}_{J}=0\right)$. The limiting cases of zero foil spacing $(L=0)$ corresponding to no electric defocusing and infinite foils spacing $(L \rightarrow \infty)$ corresponding to vacuum propagation are shown in blue and green, respectively. (b) Expanded scale plot including values of $L$ giving focusing. Data of minimum rms-extent points for $L$ leading to focusing is given in Table I.

focusing, $\sigma_{x}$ decreases with increasing rate as $\sigma_{x}$ becomes smaller until the aspect ratio eventually deteriorates. This "inertia" of inward focusing motion drives the beam beyond the critical aspect ratio where the electric force becomes larger than the magnetic force, but eventually the electric force overcomes the inward inertia, and forces the beam back to larger $\sigma_{x}$. For foil spacing $L=100 \mu \mathrm{m}$, which is near the critical value with $L=\sigma_{x i} / 1.94=$ $103 \mu \mathrm{m}$, the initial envelope is nearly in force balance and only evolves minimally. For cases with $L>100 \mu \mathrm{m}$, the magnetic force is inadequate to focus, and the electric defocusing force drives $\sigma_{x}$ to larger values than the initial value $\sigma_{x}=\sigma_{x i}$.

The limiting form solution with $L=0$ is included on the plots in Fig. 8 so the strength of the magnetic focusing relative to the extreme case with no electric defocusing force $(F=0)$ is illustrated. As discussed in Sec. IV C, the limit $L=0$ corresponds to the beam propagating through solid metal and the beam current would likely be neutralized to inhibit focusing in this case. Nevertheless, this idealization is included to illustrate that within the context of the model there are limits to the obtainable reduction in propagation distance to the focal spot as the foil spacing is reduced. In the special case with $L=0$, the beam is rapidly focused to zero rms extent $\left(\sigma_{x}=0\right)$ within the context of the paraxial model because there is no beam emittance $\left(\varepsilon_{x, \text { rms }}=0\right)$ or canonical angular momentum $\left(P_{\theta}=0\right)$. The propagation distance to focus (see Table I) for $L=0$ is consistent with Eq. (56), which gives $z_{f}=\frac{\sqrt{2 \pi / Q}}{\gamma_{b} \beta_{b}} \sigma_{x i}=$ $37.8 \mathrm{~mm}$. For the cases of foil spacings with $L>0$ in Fig. 8, finite-beam emittance $\varepsilon_{x, \text { rms }} \neq 0$ and/or canonical angular momentum $P_{\theta} \neq 0$ can further limit the minimum value of $\sigma_{x}$ achieved in addition to the balance of magnetic and electric forces to result in larger spots. If necessary, focusing can be augmented by superimposing an applied solenoid field $(\kappa>0)$ to further reduce rms-beam extents and/or increase system tuning possibilities. Lower beam kinetic energy $\left(\mathcal{E}_{b}\right)$ and larger initial beams $\left(\sigma_{x i}\right)$ will increase the effectiveness of the solenoidal field. The vacuum solution corresponding to infinite foil spacing $(L \rightarrow \infty)$ is also indicated in Fig. 8(a). In this case, the beam envelope rapidly expands off the scale of the plot because there is no net focusing and the perveance $Q$ is large.

Results in Fig. 8 suggest that if relatively long path length transport and/or beam collimation from a TNSA source is desired, then the incident beam should be focused to the value of transverse rms extent $\sigma_{x}$ desired for

TABLE I. Minimum $\sigma_{x}$ and corresponding $z$ location for select envelope solutions in Figs. 8 and 9.

\begin{tabular}{lccccccccc}
\hline \hline Foil & \multicolumn{3}{c}{\begin{tabular}{c}
\multicolumn{3}{c}{ Focal spot } \\
Spacing
\end{tabular}} & \multicolumn{3}{c}{ Figure 8: $\sigma_{x i}^{\prime}=0$} & \multicolumn{2}{c}{ Figure $9(\mathrm{a}): \sigma_{x i}^{\prime}=5 \mathrm{mrad}$} & \multicolumn{2}{c}{ Figure 9(b): $\sigma_{x i}^{\prime}=-5 \mathrm{mrad}$} \\
$L(\mu \mathrm{m})$ & $\sigma_{x}(\mu \mathrm{m})$ & $\sigma_{x} / L$ & $z(\mathrm{~mm})$ & $\sigma_{x}(\mu \mathrm{m})$ & $\sigma_{x} / L$ & $z(\mathrm{~mm})$ & $\sigma_{x}(\mu \mathrm{m})$ & $\sigma_{x} / L$ & $z(\mathrm{~mm})$ \\
\hline 100 & 188. & 1.88 & 66.6 & 121. & 1.20 & 151. & 120. & 1.21 & 22.0 \\
75 & 111. & 1.48 & 53.7 & 85.9 & 1.15 & 114.9 & 85.9 & 1.15 & 24.9 \\
50 & 58.4 & 1.17 & 45.2 & 50.3 & 1.01 & 93.5 & 50.3 & 1.01 & 25.0 \\
25 & 22.3 & 0.892 & 40.1 & 20.4 & 0.819 & 82.0 & 20.5 & 0.819 & 23.7 \\
0 & 0 & $\cdots$ & 37.8 & 20 & $\cdots$ & 78.0 & 0 & $\cdots$ & 22.7 \\
\hline \hline
\end{tabular}


transport with the foil spacing $L$ set close to the critical value to balance the magnetic focusing and the electric defocusing forces. This strategy will minimize the number of foils required and resulting beam energy losses and scattering-induced emittance growth from propagating through metallic foils with finite thickness. For nonnegligible emittance or canonical angular momentum, the foil spacing should be consistently adjusted (including any adjustment for emittance growth resulting from either scattering or nonlinear fields). Higher beam energy $\mathcal{E}_{b}$ can help reduce the number of foils while allowing penetration of more foils with minimal energy losses and scattering. However, perveance $Q$ must be maintained with increasing energy to preserve the strength of the focusing (see analysis that follows). Alternatively, if a small focal spot in $\sigma_{x}$ is desired, results in Fig. 8 suggest use of a relatively minimal foil spacing $L$ with the initial beam entering the lens with a significantly larger value $\sigma_{x}>L$ so that the inward inertia gained as the beam focuses can drive $\sigma_{x}$ to smaller values than suggested by the critical aspect ratio consistent with any finite-beam emittance and/or canonical angular momentum. The value of the beam emittance $\varepsilon_{x, \text { rms }}$ or canonical angular momentum $P_{\theta}$ can be important because it can limit ultimate focusability and therefore whether there is any advantage to using smaller foil spacing $L$ if fabrication limits allow. In applications where the small focal spot in $\sigma_{x}$ should converge on a target "downstream" of the final foil, it will likely prove necessary to provide adequate plasma neutralization for target locations for axial distances $\gtrsim L / 2$ downstream of the final foil. Without neutralization, large defocusing space-charge forces can drive rapid increases in $\sigma_{x}$ downstream of the last foil.

For simplicity, all results are shown for fixed foil spacing $L$. When small focal spots are desired, it may be beneficial to reduce beam energy losses and scattering by using larger $L$ initially, and then decrease $L$ near the spot to improve the aspect ratio as the beam is focused and maintain focusing inertia for improved final spot size. This technique can reduce the number of foils required in the longer initial stage of the focusing cycle before $\sigma_{x}$ begins reducing rapidly.

To better understand system requirements, the effect of initial beam envelope divergence $\left(\sigma_{x i}^{\prime}>0\right)$ or convergence $\left(\sigma_{x i}^{\prime}<0\right)$ as well as variations in beam perveance $(Q)$ should be evaluated. First, in Fig. 9, envelope solutions are given for the same parameters and initial conditions as in Fig. 8 except with the zero initial envelope angle $\sigma_{x i}^{\prime}=0$ replaced by small diverging $\left(\sigma_{x i}^{\prime}>0\right)$ and converging $\left(\sigma_{x i}^{\prime}<0\right)$ initial angles. Corresponding data for minimum rms-extent points is given for most foil spacings $L$ in Table I. Figure 9(a) demonstrates that even a relatively small initial beam divergence $\left(\sigma_{x i}^{\prime}=5 \mathrm{mrad}\right)$ can lead to dramatically longer propagation distances in cases where the lens is intended to focus to a small spot. This occurs because the initial magnetic focusing force becomes
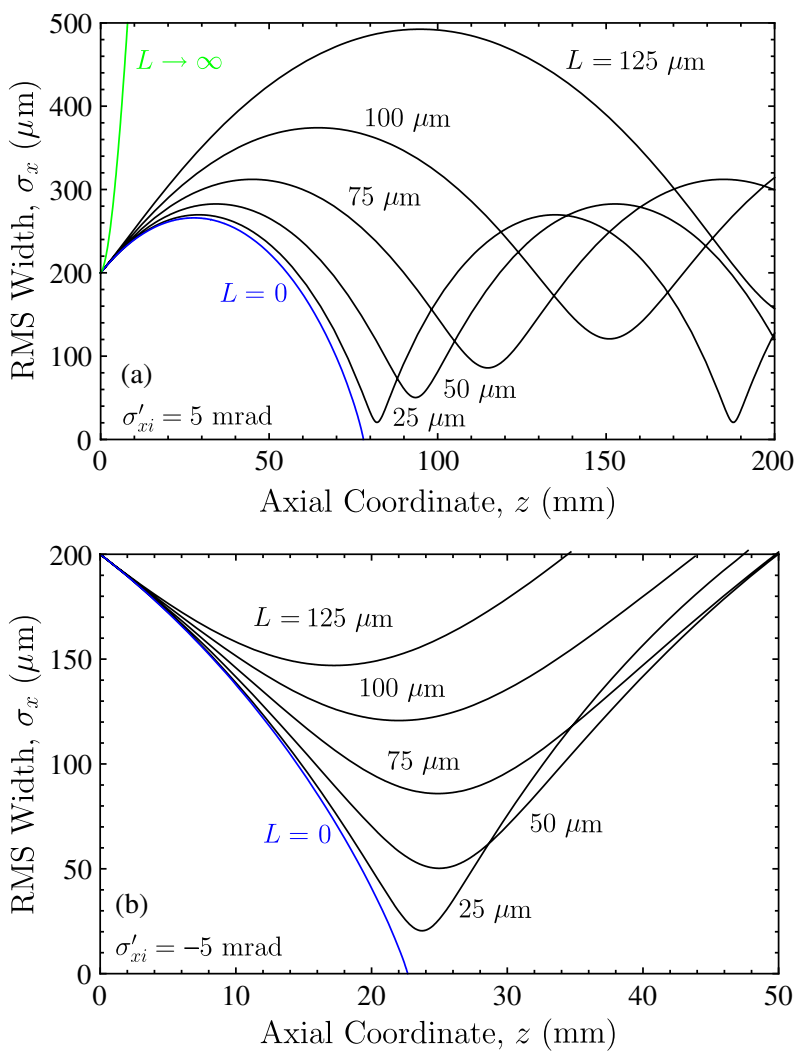

FIG. 9. Influence of initial envelope divergence (a) with $\sigma_{x i}^{\prime}=$ $5 \mathrm{mrad}$ and convergence (b) with $\sigma_{x i}^{\prime}=-5 \mathrm{mrad}$ is illustrated by repeating the envelope solutions illustrated in Fig. 8 with $\sigma_{x i}^{\prime}=0$ with the specified values of $\sigma_{x i}^{\prime} \neq 0$. Data on minimum rms-extent points for some values of $L$ is given in Table I.

weaker as the beam expands. Because the magnetic focusing term varies as $\sim 1 / \sigma_{x}$, a diverging beam extends the propagation length where the force is weak even though the aspect ratio $\sigma_{x} / L$ is driven into a more favorable regime with increasing $\sigma_{x}$. Long propagation lengths may require too many foils to be practical. In the limiting case with $L=0$, the propagation distance to focus (see Table I) is consistent with Eq. (55) which predicts a factor of $\exp \left[\sigma_{x i}^{\prime 2} /\left(2 Q_{\text {eff }}\right)\right]\left\{1+\operatorname{erf}\left[\sqrt{\sigma_{x i}^{\prime 2} /\left(2 Q_{\text {eff }}\right)}\right]\right\}=2.06$ increase for $\sigma_{x i}^{\prime}=5 \mathrm{mrad}$ in the propagation distance to the limiting pinch relative to the case with $\sigma_{x i}^{\prime}=0$.

Conversely, Fig. 9(b) shows that even relatively small initial envelope convergence $\left(\sigma_{x i}^{\prime}=-5 \mathrm{mrad}\right)$ is beneficial because the converging beam establishes inertia in the right direction to reduce the propagation length where $\sigma_{x}$ is larger before the magnetic focusing force acts strongly as $\sigma_{x}$ becomes smaller. Thus, initial envelope convergence can significantly reduce the total propagation distance and number of foils necessary to focus the beam to a limiting spot. In both cases of diverging and converging initial envelopes, the final spot size is modestly reduced relative to the case with $\sigma_{x i}^{\prime}=0$ (see minimum rms-extent data in Table I). This occurs because the envelope is driven down 
with enhanced "inertia." For the diverging case $\left(\sigma_{x i}^{\prime}>0\right)$, this enhancement occurs because the rapid decrease in $\sigma_{x}$ starts from larger values of $\sigma_{x}$ after focusing eventually turns the beam. For the converging case, the effect occurs because the initially converging envelope contributes to the inward inertia. For the converging case $\left(\sigma_{x i}^{\prime}<0\right)$, the reduction in spot size is a bonus on top of the significantly smaller propagation length to the spot. For the diverging beam, the benefit of reduced spot size is likely to be insufficient to offset the penalty of much longer propagation lengths required to the spot. In the limiting case with $L=0$, the propagation distance to focus (see Table I) is consistent with Eq. (55) which predicts a reduction factor of $\exp \left[\sigma_{x i}^{\prime 2} /\left(2 Q_{\text {eff }}\right)\right]\left\{1-\operatorname{erf}\left[\sqrt{\sigma_{x i}^{\prime 2} /\left(2 Q_{\text {eff }}\right)}\right]\right\}=0.599$ for $\sigma_{x i}^{\prime}=-5 \mathrm{mrad}$ in the propagation distance to the limiting pinch relative to the case with $\sigma_{x i}^{\prime}=0$.

These results show that it is critical to control initial envelope angle $\sigma_{x i}^{\prime}$ of the beam incident on a stacked foil lens when small focal spots are desired. A superimposed focusing solenoid $(\kappa>0)$ may prove attractive to counteract the effects of any (planned or unplanned) initial envelope divergence because the solenoid can be tuned to change the initial envelope angle as well as further augment the initially weak focusing strength in the lens where $\sigma_{x}$ is larger. Results also suggest that a thin-foil lens is well suited to a two-stage focusing system where a conventional optic forms the first stage and the thin-foil lens forms the second stage. The first optic can reduce the beam extent for stronger focusing, provide an initial converging envelope angle in the foil lens to limit propagation distance to the minimum spot in the thin-foil lens, and provide system tunability. The target at the focal spot can be incorporated into the structure of the foil lens.

The influence of varying the beam perveance $Q$ is illustrated in Fig. 10 by repeating the $L=50 \mu \mathrm{m}$ envelope solution in Fig. 8 for several values of $Q$ about the reference case with $Q \equiv Q_{0}=8.13 \times 10^{-3}$. Larger $Q$ increases the strength of the focusing leading to substantially shorter propagation distance to the spot. Notice that the minimum value of $\sigma_{x}$ at the focal spot has insignificant variation in $Q$. This is because, for this foil spacing, the change in focusing angle (and the focusing inertia) due to the perveance variation is insufficient to significantly alter the minimum value of $\sigma_{x}$ at the focal point. Because $Q \propto \lambda /\left(\gamma_{b}^{3} \beta_{b}^{2}\right) \propto I /\left(\gamma_{b} \beta_{b}\right)^{3}$ [see Eq. (42)], increasing beam kinetic energy $\mathcal{E}_{b}$ (i.e., larger $\gamma_{b} \beta_{b}$ ) reduces the beam perveance, unless accompanied by appropriately larger beam current $I$ to compensate for the larger value of $\gamma_{b} \beta_{b}$ with increasing energy. Although increasing beam energy aids in reducing beam energy attenuation and scattering in the foils as well as allowing larger foil spacing $L$ (i.e., improved aspect ratio) for beam focusing, higher current is simultaneously desirable to maintain the value of the perveance $Q$ and thereby the strength of the focusing for a short beam propagation distance to the focal spot.

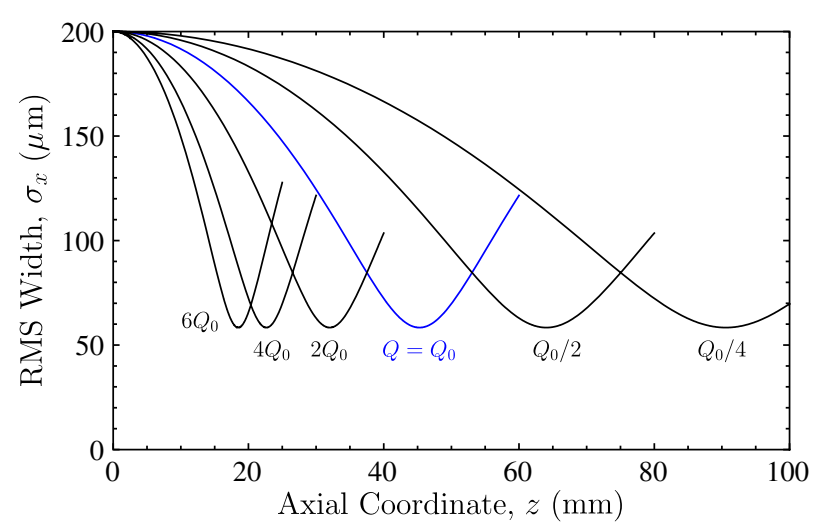

FIG. 10. Influence of varied beam perveance $Q$ is illustrated by repeating the envelope solution in Fig. 8(b) with $L=50 \mu \mathrm{m}$ and $Q \equiv Q_{0}=8.13 \times 10^{-3}$ (blue) to the values of $Q$ as labeled.

To better characterize the likely range of variations in envelope solutions due to possible changes in the form factor $F$ associated with possible evolution in the beam radial charge profile, we repeat the solutions in Fig. 8(b), which are calculated for a Gaussian-chargedensity beam, for an rms-equivalent, uniform-chargedensity beam. These solutions are contrasted in Fig. 11. Because all differences are contained in the form factor when emittance and angular momentum are negligible $\left(\varepsilon_{x, \mathrm{rms}}=0=P_{\theta}\right)$, we employ $\sigma_{x}=\left\langle x^{2}\right\rangle_{\perp}^{1 / 2}=r_{b} / 2$ for rms equivalency and replace the Gaussian-beam form factor expression in Eq. (49) with the uniform-beam case setting the aspect ratio as $r_{b} / L=2 \sigma_{x} / L$. Note that differences between the Gaussian and uniform-beam evolution are smaller in foil spacing cases where the beam is transversely focused strongly, and differences are larger when the initial beam is close to radial force balance between magnetic focusing and electric defocusing forces. This is not surprising because form factor

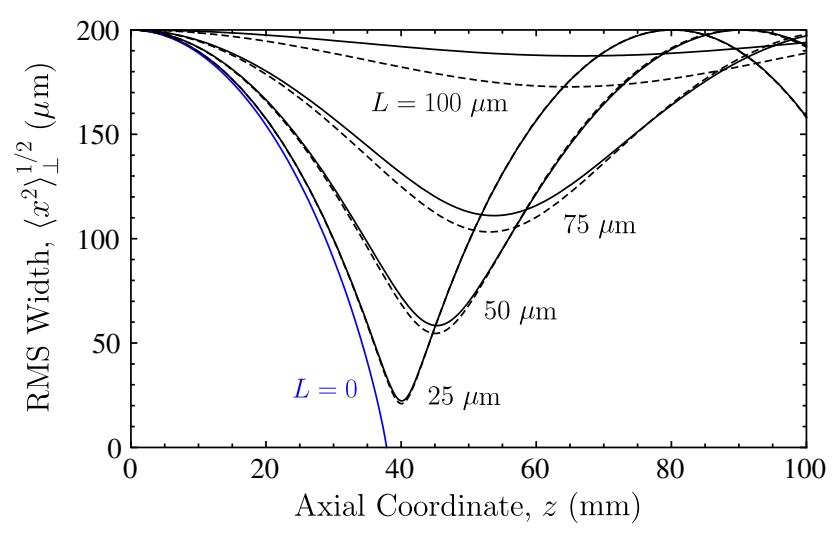

FIG. 11. Influence of different form factor $F$ on the envelope evolution is illustrated by repeating the envelope solutions in Fig. 8(b) (solid curves) for Gaussian-beam charge density with an rms-equivalent uniform-charge-density beam. For $L=0$ (blue) the solutions exactly overlay. 
differences are largest close to aspect ratios where the balance occurs (see Fig. 6). Strong focusing cases (small foil spacing) result in the envelope evolving only a small fraction of the focusing cycle in aspect-ratio regions where the form factor differences are most appreciable. However, in the near balanced cases, the full focusing cycle evolves in the range of largest form factor differences. Furthermore, one might expect more sensitivity to deviations in the electric defocusing term in situations where the magnetic focusing and electric defocusing are in near balance. In the fully self-consistent beam evolution, the form factor will likely evolve during the focusing cycle due to evolution in the beam charge density. In cases with applied focusing $(\kappa \neq 0)$ and strong spacecharge, evolution outside of self-similar form may be relatively small because strong Debye screening of the linear focusing force should result in a beam charge profile which is nearly uniform in the core out to the radial edge where the density rapidly drops to small values $[28,32]$. In such situations, little evolution in the form of $F$ should be possible. Conversely, in cases with no applied focusing $(\kappa=0)$, the form of the profile may evolve more strongly from the initial form in response to self-magnetic focusing and electric defocusing forcesparticularly if the initial profile is a consequence of neutralization physics and neutralizing electrons are blocked as the beam enters the foils. Regardless, differences in form factor from one smooth charge-density profile to another, reasonably similar, rms-equivalent smooth profile will likely impact evolution less than the cases illustrated in Fig. 11 which highlight differences resulting between fixed form Gaussian and uniform-beam form factors which are for beams with very different radial charge-density profiles $\rho_{b}(r)$ (see Fig. 3). However, simulations (see Sec. VI) suggest that situations where pronounced halo develops may exacerbate form factor differences. If desired, form factors for other smooth charge-density profiles can be calculated explicitly using Eq. (43) in either the continuous approximation or with full $z$ variation. Simulations or higher level theory can be applied to model the evolution more consistently if more accuracy in $F$ is necessary. In warm beam evolution with non-negligible emittance, one might anticipate more differences than those contained in the form factor due to the emittance term which is neglected in the present analysis. The emittance will self-consistently evolve in response to nonlinear forces. Proper ordering of such effects is necessary to avoid spurious perceptions of enhanced model accuracy by improvements in the form factor term without accounting for the emittance or vice versa. The efficiency of the envelope model as approximated with a fixed form factor allows rapid evaluation of effects to provide insight. At high levels of model detail, it is likely that full self-consistent simulations (see Sec. VI) are most appropriate.

\section{PARTICLE-IN-CELL SIMULATIONS}

This section presents a comparison of the envelope theory with particle-in-cell simulations using the WARP code [47-49]. Attention is restricted primarily to simulations aimed at verifying the envelope-model predictions and the departure from those results in cases where significant beam halo is generated, the beam pulse length is short, and/or and the beam under study is that of a finite-width energy slice of a much broader-energy-spectrum (and correspondingly higher-total-current) beam. For illustrative purposes, system parameters corresponding to the examples in Sec. V are employed except where noted for purposes of simulation efficiency and/or investigating lesser degrees of idealization. Emphasis is placed on physics and the relative importance of effects. The simulations do not constitute a model of laboratory experiments which may have many competing effects occurring simultaneously. This is part of a larger ongoing simulation campaign to explore the physics of self-pinching proton and ion beams including many additional effects: combined beam energy and transverse velocity spreads, an initially converging or diverging beam envelope, scattering by the foils, effects of electrons introduced from the source or emitted from the foils, fully electromagnetic and 3D fields, etc. Results of this more general study will be described in a separate paper.

We simulate only initially perfectly collimated proton beams, with no transverse velocity and no longitudinal velocity spread. This corresponds to a beam distribution with zero initial envelope angle $\left(d\left\langle x^{2}\right\rangle_{\perp}^{1 / 2} / d t=0\right.$ at time $t=0)$ and zero rms emittance $\left(\varepsilon_{x, \mathrm{rms}}=0\right.$ at $\left.t=0\right)$. We employ a quasistatic and axially symmetric field approximation with infinitesimally thin, perfectly conducting foils with no particle scattering within the foils. The simulations follow the full self-consistent Vlasov evolution of the distribution from the initial condition. Thus, they serve to check the validity of assumptions made in the simplified envelope model, including modeling the evolution in the charge density as self-similar, averaging over the rapid $z$ variation of the defocusing electric field (i.e., the continuous defocusing approximation of the form factor $F$ ), neglect of corrections to the magnetic focusing terms resulting from envelope convergence, and the neglect of emittance evolution for a beam with negligible initial emittance.

Our simulation geometry is axisymmetric cylindrical $(r-z)$. We use quasistatic electrostatic and magnetostatic approximations $\left(\nabla \cdot \mathbf{E}=\rho / \epsilon_{0}, \nabla \times \mathbf{B}=\mu_{0} \mathbf{J}\right)$ to calculate the electric and magnetic fields on a uniform $r-z$ grid. The quasistatic field approximation is adequate for the relatively low beam energies of the present study; comparison of quasistatic and fully electromagnetic simulations shows no qualitative differences even for much higher-energy (moderately relativistic) ion beams. The problem setup is that of an axially uniform column of 
protons, with a radial density profile that is either constant out to a cutoff at $r=r_{b}$ or a Gaussian, as described in Sec. II. The simulation domain extends radially from $r=0$ to $r=3 r_{b}=6 \sigma_{x}$. The initial particle loading in $z$ is uniform, and is also regular in $r$, with spacing uniform in $r^{2}$ for the constant-density case, or uniform in $R^{2}$ out to $R=r_{b}=2 \sigma_{x}$ for the Gaussian distribution, with $R$ related to the actual radius by the relation

$$
R=\sigma_{x}\left[-2 \ln \left\{-\left(\frac{r}{2 \sigma_{x}}\right)^{2}\left[1-\exp \left(\frac{r_{\text {max }}^{2}}{2 \sigma_{x}^{2}}\right)\right]\right\}\right]
$$

which renders the distribution Gaussian out to a cutoff at $r_{\max }=3.5 \sigma_{x}$.

We consider two types of simulations. The first has a short-length box with conducting walls at the ends to simulate the space between two foils as part of an infinitely long beam. The particle boundary conditions are periodic in $z$ and absorbing in $r$. The boundary conditions on the electric field are conducting at the ends and the radial edge of the grid, and for the magnetic field, periodic at the ends and "conducting" (vector potential components zero) at the radial edge. We refer to these simulations as "infinite beam." For infinite-beam simulations, we calculate the rms-transverse beam extent $\left\langle x^{2}\right\rangle_{\perp}^{1 / 2}$ averaged over the full axial domain in $z$. This improves statistics and results in relatively small error because axial variation in transverse averages are typically small within one foil gap.

The second simulation type has a finite beam, (typically) many conducting foils, and a simulation domain that is a moving window that extends axially $4 r_{b}=8 \sigma_{x}$ beyond the ends of the initial-length beam (to ensure insensitivity to axial boundary conditions). At the radial edge of the grid, the field boundary conditions are conducting and the particle boundary condition is absorbing. With such an extended domain, we find no noticeable difference among simulations with periodic, Neumann, or Dirichlet boundary conditions on the potentials at the axial grid ends. No particles reach the ends of the moving window during the simulations. We refer to these simulations as "finite beam." For this type of simulation we calculate $\left\langle x^{2}\right\rangle_{\perp}^{1 / 2}$ averaged over the central portion of the axial extent of the beam (1/16 of the beam length) because we find that the beam ends evolve differently than the beam interior due to rapid axial self-field variation at the beam ends. These simulations have a much larger simulation domain and so require substantially more computational time than the infinite-beam simulations described above.

The infinite-beam simulations, being computationally less demanding, are better suited to probe resolution issues and are convenient for comparison with the theory (which also assumes a long, unbunched beam), whereas the finitebeam simulations are necessary to probe effects from finite pulse duration.

In order to reduce simulation time, we use a current density that is 6 times the base value employed in Sec. V, that is with perveance $(Q \propto I) Q=6 Q_{0}=4.88 \times 10^{-2}$. The envelope model (see Fig. 10) predicts little difference in spot size due to the increased perveance, but a significantly shorter propagation distance to the focal point. The resulting savings in simulation time enables parametric simulations to be carried out in the finite-beam case at sufficient resolution and statistics. In all cases, we consider a beam which initially has $\sigma_{x}=200 \mu \mathrm{m}$ for Gaussian loading, or the rms equivalent $r_{b}=400 \mu \mathrm{m}$ for uniform loading. Except as noted, in all cases presented there are $n_{r}=64$ radial simulation cells and the number of axial simulation cells is chosen so that there are eight cells between foils. Grid spacings are chosen such that the grounded thin foils lie on $z$-mesh points. (This restriction is not necessary; WARP's embedded-boundary capability allows conductors of arbitrary thickness to be placed with boundaries between mesh points.) The initial number of particles per cell, averaged over beam-containing cells with $r \leq \sigma_{x}$, is 296 for the infinite-beam simulations and 37 for the finite-beam simulations. However, the statistics are not as dissimilar between these cases as these numbers might suggest, because the magnetic field for the finitebeam simulations is a response to the particles in many more cells (over a range in $z$ comparable to the beam radius), versus just over the eight cells between foils for the infinite-beam simulations. Convergence studies both with respect to particle number (in both the infinite and finite-beam setups) and (increased) spatial resolution indicate that the quantities analyzed here are well converged for the stated parameters. Test simulations show insensitivity of results to the choice of radial grid extent, rendering idealizations made in boundary conditions for the magnetic field at the radial edge of the grid irrelevant.

We perform infinite-beam simulations for beams with Gaussian radial density profiles and foil spacings $L=$ $25,50,75$, and $100 \mu \mathrm{m}$, as well as a "zero-spacing" $(L=0)$ case with the electric-field calculation turned off. Results for evolutions in rms $x$ width $\left\langle x^{2}\right\rangle_{\perp}^{1 / 2}$ and rms-edge emittance $\varepsilon_{x}$ [Eq. (47)] are shown in Fig. 12 (solid curves), along with the corresponding theory results (dashed curves) from the envelope model calculated in the continuous defocusing approximation with consistent perveance (i.e., $Q=6 Q_{0}$ ). Results for one uniform-density case with foil spacing $L=50 \mu \mathrm{m}$, and both uniform and Gaussian cases with no foils (vacuum case; approximate due to the radial field boundary conditions) are also shown. The simulations and envelope-model results for the rms $x$-width in Fig. 12(a) agree reasonably well, particularly considering that the simulation has a number of complications not considered in the theory: (i) an evolving radial density profile [hence the $F$ form factor introduced in Eq. (43) is nonconstant; although we expect from the comparisons in Fig. 11 that this should be a small effect over a range of profile shapes], (ii) a focus force that varies in $z$ versus being approximated by its average; and 

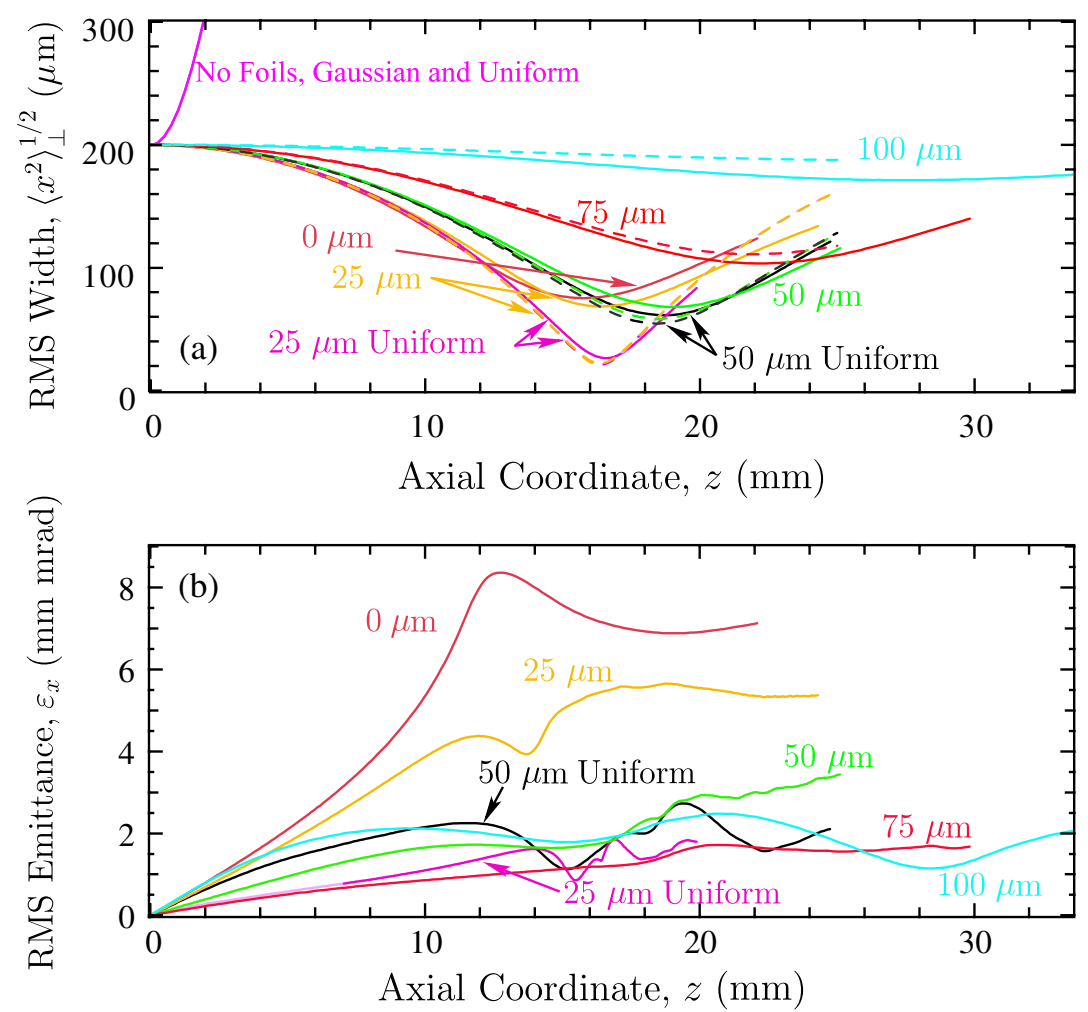

FIG. 12. (a) rms $x$-envelope $\left(\left\langle x^{2}\right\rangle_{\perp}^{1 / 2}\right)$ and (b) rms-edge emittance $\left(\varepsilon_{x}=4 \varepsilon_{x, \text { rms }}\right)$ evolution for infinite-beam WARP simulations (solid curves) with various foil spacings $L$ as indicated. Dashed curves are predictions from the envelope model with zero emittance. Simulations and envelope-model results are for Gaussian initial radial density profiles, except for indicated cases for simulations with initial uniform density. Simulation results for no foils with Gaussian and uniform initial profiles lie almost on top of one another. The $L=25 \mu \mathrm{m}$ simulation results for the uniform and Gaussian initial density profiles also are nearly coincident, and the corresponding $L=25 \mu \mathrm{m}$ envelope-model result is close to the simulations.

(iii) finite emittance growth from zero in the simulations due to nonlinear self-fields. We note that the two curves for the Gaussian and uniform cases with no foils (vacuum) lie on top of one another. The corresponding evolution of the rms-edge emittance is shown in Fig. 12(b) for all cases in Fig. 12(a) except the vacuum cases (which are excluded because the beams quickly hit the simulation boundaries which corrupts the emittance measure due to the absorbing
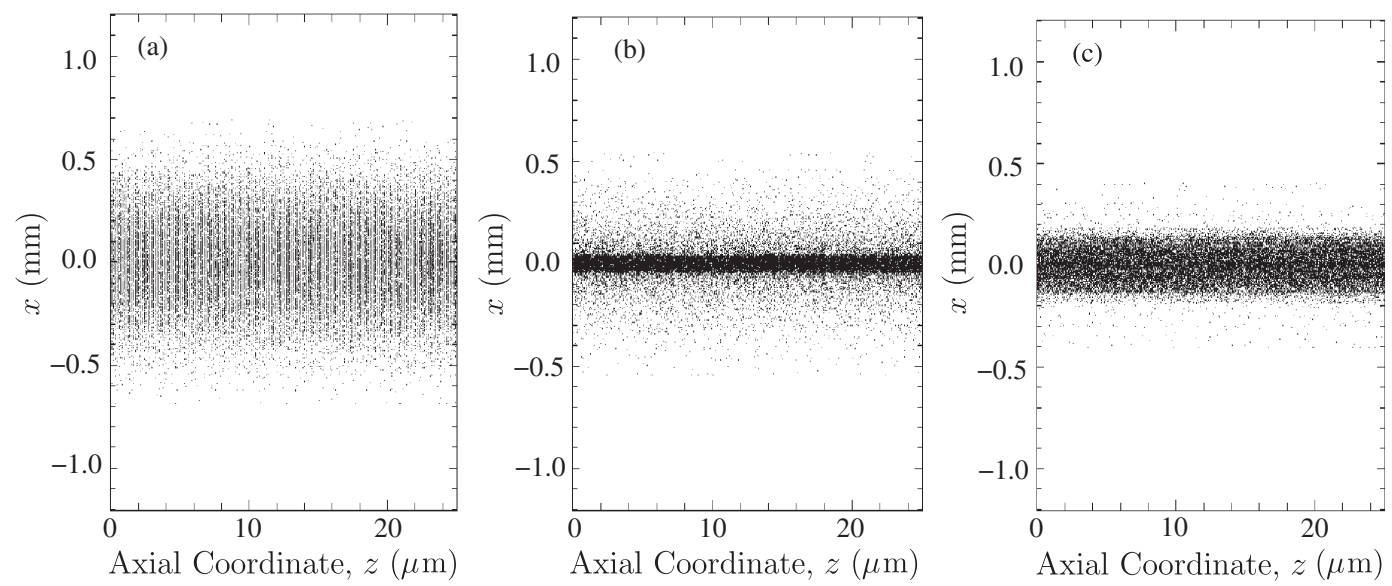

FIG. 13. Particle scatter plots for an infinite-beam WARP simulation with $L=25 \mu \mathrm{m}$ foil spacing at times $t=$ (a) 19 , (b) 315 , and (c) $430 \mathrm{ps}$ (corresponding to axial propagation lengths of $0.84,13.9$, and $18.9 \mathrm{~mm}$ through the foil stack). Plot (b) corresponds approximately to the minimum rms $x$ width of the dense core of particles seen in the plots. The horizontal axis of the plots represents the distance from the left-hand simulation boundary to the right-hand boundary (i.e., between foils). Plot (a) may appear to have axial density oscillations; however, this is an artifact resulting from beating between the uniformly spaced initial loading of particles and the graphic resolution. 

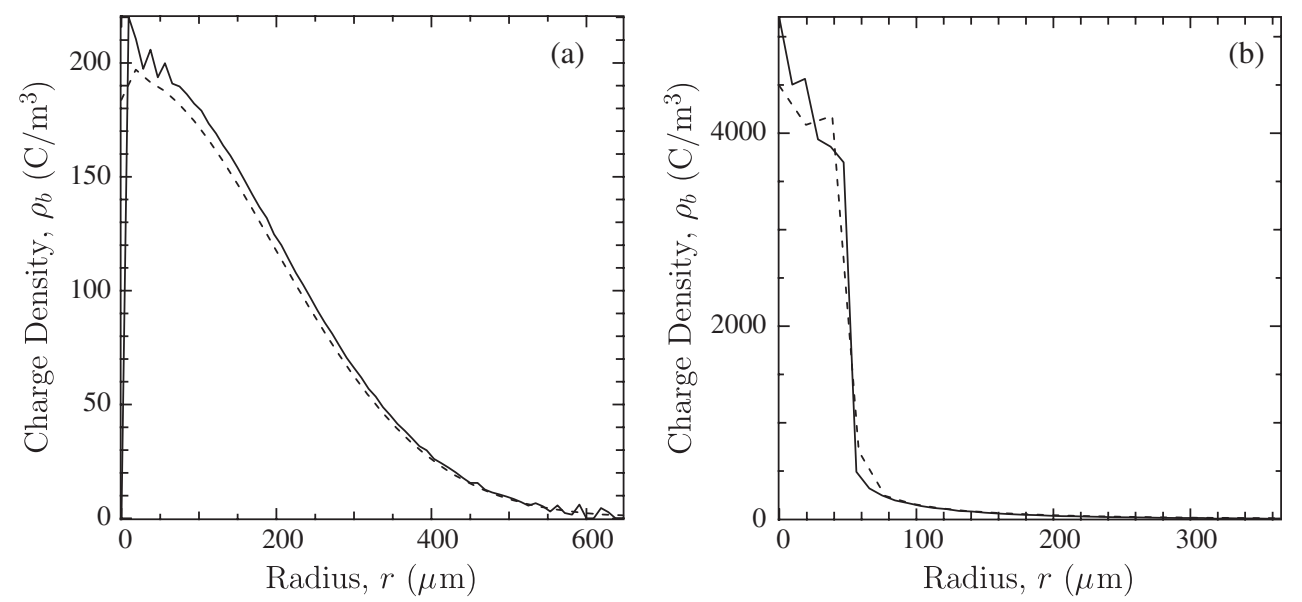

FIG. 14. Radial charge-density variation for infinite-beam WARP simulations with $L=25 \mu \mathrm{m}$ foil spacing (a) early in run, at $t=19 \mathrm{ps}$, and (b) at $t=315 \mathrm{ps}$. Dashed curves are for resolution $n_{r} \times n_{z}=64 \times 8$; solid curves are for $n_{r} \times n_{z}=128 \times 16$.

boundary conditions). Particle scatter plots (see Fig. 13) suggest that the relatively large discrepancy in the minimum envelope size in the $L=0$ and $L=25 \mu \mathrm{m}$ cases can be attributed to the development of an appreciable halo; the core of the beam does indeed compress to an $x$ width that is quite a bit smaller than the rms-envelope size. We see the phenomenon in another way in Fig. 14, where we plot the (charge) density versus radius near the beginning of the run (time $t=19 \mathrm{ps}$ ) and at a time where the core beam distribution is near its minimum rms $x$ width $[t=315 \mathrm{ps}$, the same time as the plot in Fig. 13(b)]. The shape of the charge distribution when the core is highly compressed changes appreciably, is distinctly non-Gaussian, and has a significant radial tail (the halo). We remark that Fig. 14 contains plots at both the standard resolution $\left(n_{r} \times n_{z}=\right.$ $64 \times 8)$ and doubled resolution $(128 \times 16)$, to support our conclusion that the profile shape evolution is not an artifact associated with low resolution. We also note that the

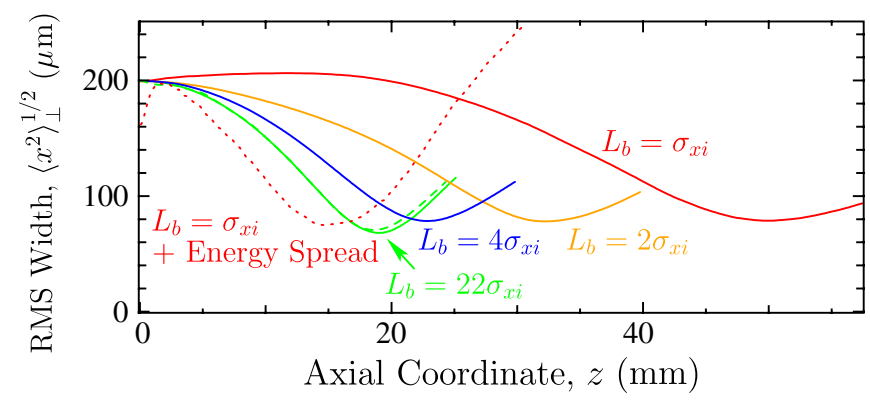

FIG. 15. Envelope evolution for finite-length beam WARP simulations with various initial beam lengths $L_{b}$ as indicated (color coded) and fixed $L=50 \mu \mathrm{m}$ foil separation. Solid curves show the rms-transverse extent averaged over the middle eighth of the axial extent of the beam (middle quarter for $L_{b}=\sigma_{x}$, for better statistics). The green dashed curve gives the corresponding result for an "infinite-beam" simulation with the same foil separation. The dotted curve is for an $L_{b}=\sigma_{x i}$ simulation with a broad coherent energy spectrum as specified in the text. minimum rms width for the corresponding uniform-density case is about half that for the Gaussian case, and much closer to theory; this is associated with considerably less halo formation in the scatter plots. The envelope-model (theory) calculations show little difference in minimum spot size between uniform and Gaussian radial shapes, suggesting an insensitivity to the radial shape; but that insensitivity should not be expected in cases where a substantial halo is formed, as the halo particles see substantially weaker fields than the main distribution and therefore focus over a longer distance (a phenomenon that is clearly evident in movies made from a sequence of scatter plots). The Gaussian-beam simulations with larger foil separations also develop substantial halo, but the halo makes relatively less difference in those cases because the radial core of the beam is not compressing as much. The more significant halo in the Gaussian case relative to the uniform-density case can likely be attributed to the significant fraction of the beam that is in a region where the net focusing force is increasing significantly more slowly than linear in (or even decreasing with) radius. The same observation is likely responsible for the larger rms-emittance growth in the Gaussian cases: halo particles contribute more to the emittance.

Comparison of the initially uniform and Gaussian simulations suggest that the differences noted above between the focal spot sizes in the simulation and envelope-model results would likely be substantially reduced for a measure of spot size that is less sensitive to halo-for example, mean radius rather than rms $x$ width, or only considering the distribution function above sufficiently large threshold (as may be characteristic of experimental diagnostics). However, the results also suggest that, depending on the measurement technique, the observed spot may vary significantly with the tail structure of the initial distribution, which in turn may vary depending on how the initial beam is formed. 
We have also performed a series of finite-beam simulations with varying initial axial beam length $L_{b}$ and fixed $L=50 \mu \mathrm{m}$ foil spacing. The obtained rms-width evolutions are shown in Fig. 15 (solid curves), along with, for comparison, the corresponding infinite-beam simulation (dashed curve). In order to minimize clutter, we do not show the corresponding envelope-model curve for here, but from Fig. 12(a) it can be seen that the $L=50 \mu \mathrm{m}$ infinite-beam simulation is also an excellent proxy for the long pulse envelope model. The results show a notsurprising result: as the initial beam length $L_{b}$ is made shorter relative to the initial rms width $\sigma_{x}=\sigma_{x i}$, the focal distance gets longer. The departure for $L_{b}=2 \sigma_{x i}$ and $L_{b}=\sigma_{x i}$ is substantial. The minimum focal spot, however, does not change much as the beam length is varied (in contrast to the change we find with varying foil spacing). These results are easily understood: as the beam length decreases, the focusing magnetic field, which is sensitive to the current integrated over an axial distance of order of the characteristic transverse beam radius (in all cases at the initial time, many foil spacings) decreases, while the defocusing electric field, which is sensitive only to the charge density between two foils, is virtually unchanged near the beam's axial center. Hence, the relative balance between magnetic focusing and electric defocusing is shifted away from focusing. On the other hand, once the beam starts to compress, its length becomes longer relative to its radius, and hence the magnetic pinch force becomes closer to the infinite-beam limit-i.e., it increases faster with decreasing radius than does the electric defocusing.

As noted previously, the parameters chosen in Sec. V are long pulse idealizations of a recently performed near-term test of the self-focusing concept using a TNSA produced proton beam at the PHELIX facility [10]. The PIC simulations in this section employ the same basic system parameters with higher current to reduce numerical modeling time. Laboratory laser-produced proton beams are likely to have initial distribution properties that significantly deviate from the idealizations made. Moreover, distribution properties are likely to vary significantly as techniques employed evolve (e.g., use of curved emission foils to control incident envelope divergence). Although techniques exist to produce relatively monochromatic proton beams with short pulse lasers [40-44], in typical TNSA produced proton beams the coherent beam energy spectrum is broad with particle counts decaying nearly exponentially with energy [10,13-16]. For the PHELIX facility, the variation of the TNSA produced proton distribution $(f)$ in coherent energy for the situation outlined in Sec. V can be roughly modeled by $f \propto \exp \left(-\mathcal{E}_{b} / \mathcal{E}^{*}\right)$ (i.e., a half-Gaussian in proton forward velocity $\beta_{b} c$ ) with $\mathcal{E}^{*}=4.23 \mathrm{MeV}$ [10]. For present purposes, the constant of proportionality in $f$ is fixed by requiring that the total proton current in a $1-\mathrm{MeV}$-wide energy window about $10 \mathrm{MeV}$ equals $6 \times 400.5$ A to correspond to the monoenergetic simulations analyzed above.
There are two qualitative effects that one might expect resulting from such a TNSA-like energy spectrum: on the one hand, the protons in the energy slice are spread in axial velocity over the extent of that slice, which, particularly for the short axial length beams characteristic of the actual experiment, might be expected to substantially decrease magnetic focusing due to axial spreading of the beam as it propagates through the foil system. On the other hand, there are additional protons in neighboring energy slices which contribute to the focusing magnetic field (but, as a result of the presence of the foils, not the electric field) at the position of the $10 \mathrm{MeV}$ slice. Less energetic protons will be more numerous and can have a significant contribution to the magnetic field. To illustrate the competition of these effects, we perform an additional simulation with a beam with the specified coherent energy distribution representing TNSA experiments with PHELIX. The initial beam length is $L_{b}=$ $\sigma_{x i}$, i.e., the same as for the shortest monoenergetic case (solid red curve) shown in Fig. 15. The result in Fig. 15 (dotted red curve) shows that the focal distance is reduced by a factor of about 3 compared to the monoenergetic case, while the minimum rms width is almost the same. From this, we conclude that the beneficial effect of neighboring energy contributions in a more realistic TNSA distribution likely outweighs the deleterious effect of the spread of energy within the $10 \mathrm{MeV}$ window. More detailed evaluation of particular experiments will likely require modeling of processes involved in producing the proton beam. An ongoing simulation program is carrying out such simulations including numerous simultaneous effects.

\section{CONCLUSIONS}

An envelope model has been derived to efficiently analyze the evolution of an unbunched beam propagating through a lens formed from thin, stacked metallic foils. The model accounts for beam-generated magnetic focusing forces and electric defocusing forces in the stacked foil structure as well as forces from a possibly superimposed applied solenoidal magnetic field for additional focusing and thermal defocusing associated with finite-beam emittance (phase-space area). PIC simulations with a lower degree of idealization were applied which confirm the envelope model and illustrate how results can deviate in strong focusing situations where some initial distributions with radial tails in the density profile can generate strong halo, and when the axial pulse length becomes sufficiently short to result in attenuation of magnetic focusing strength, and when coherent energy spread becomes large. Analytic analysis and simulations show that provided large halo is not generated due to the form of the initial distribution, the envelope model should be relatively insensitive to the radial structure of the transverse charge-density profile of the beam when interpreted in an rms-equivalent sense. The envelope model was applied to obtain a simple criterion for the required foil spacing to have the beam-generated 
magnetic focusing force be larger than the beam-generated electric defocusing force-which is a necessary condition to allow passive focusing. The strength of the focusing was also characterized in terms of a beam perveance parameter. Results showed that foil spacings on order of the desired transverse rms-beam extent are acceptable for focusing either for beam collimation/transport or pinching a highintensity beam to a small transverse beam spot. This is an encouraging result because it allows minimal beam propagation distance through the foils to reduce materials and undesired beam energy losses and scattering. It was found that it is critical to control any initial envelope divergence in applications where a small beam spot is desired in short propagation lengths. Illustrative examples based on the envelope model show that long pulse idealizations of laser-produced proton beams with modest parameters can lead to strong pinch focusing. Simulations confirm the basic effect under less idealized conditions with short pulse length and a broad (exponential) energy spectrum, suggesting that proof-of-principle experiments are possible if initial beam envelope divergence can be limited. Successful demonstrations can help motivate further experiments for heavy ion beams for applications such as the demanding focusing requirements for the $\mathrm{X}$-target in heavy ion fusion [6]. Follow-up studies are clarifying the influence on focusing properties due to finite scattering within the foils [17] and combined effects under likely laboratory conditions in near-term applications. Proof-of-principle proton focusing experiments have recently been carried out and will be detailed in a future report.

\section{ACKNOWLEDGMENTS}

The authors wish to thank B. G. Logan (LBNL) for suggesting the $\mathrm{X}$-target application of thin-foil focusing which motivated this research, and J. Barnard (LLNL), F. Bieniosek (LBNL), A. Faltens (LBNL), A. Friedman (LLNL), M. Roth (TU-Darmstadt), and G. Schaumann (TU-Darmsdadt) for useful discussions. The WARP PIC simulations were supported by D. Grote (LLNL) and J.L. Vay (LBNL). This research was performed under the auspices of the U.S. Department of Energy at the Lawrence Livermore and Lawrence Berkeley National Laboratories under Contracts No. DE-AC52-07NA27344 and No. DEAC02-05CH11231.

\section{APPENDIX A: APPROXIMATE ELECTRIC-FIELD SOLUTION FOR LARGE ASPECT RATIOS}

Denote the characteristic transverse scale of the beam charge density $\rho_{b}(r)$ as $r_{\perp}$. Assume that the foils are closely spaced relative to $r_{\perp}$ and introduce a small parameter $\epsilon=L / r_{\perp} \ll 1$. Anticipate that the solution to the Poisson equation (16) is characterized by axial scale lengths in $z$ being small compared to radial scale lengths in $r$ by $\Theta(\epsilon)$. Introduce a scaled variable $\mathcal{R}=\epsilon r$, so that scale lengths in $\mathcal{R}$ and $z$ are comparable. Note that the radial scale length of $\rho_{b}(r)$ is $r_{\perp}=L / \epsilon$ and denote

$$
\rho_{b}(r)=\tilde{\rho}_{b}(\mathcal{R})
$$

Then the axisymmetric beam Poisson equation (16) can be expressed as

$$
\epsilon^{2} \frac{1}{\mathcal{R}} \frac{\partial}{\partial \mathcal{R}}\left(\mathcal{R} \frac{\partial}{\partial \mathcal{R}}\right) \phi_{g}+\frac{\partial^{2} \phi_{g}}{\partial z^{2}}=-\frac{\tilde{\rho}_{b}(\mathcal{R})}{\epsilon_{0}}
$$

with $\phi_{g}(\mathcal{R}, z= \pm L / 2)=0$. We construct a solution to Eq. (A2) in a power series in $\epsilon^{2}$ as

$$
\phi_{g}(\mathcal{R}, z)=\sum_{n=0}^{\infty} \epsilon^{2 n} \phi_{n}(\mathcal{R}, z) .
$$

Inserting Eq. (A3) in Eq. (A2) obtains to leading order in $\epsilon$,

$$
\frac{\partial^{2}}{\partial z^{2}} \phi_{0}(\mathcal{R}, z)=-\frac{\tilde{\rho}_{b}(\mathcal{R})}{\epsilon_{0}},
$$

subject to $\phi_{0}(\mathcal{R}, z= \pm L / 2)=0$, and for higher orders in $\epsilon$,

$$
\begin{aligned}
\frac{\partial^{2}}{\partial z^{2}} \phi_{n}(\mathcal{R}, z) & =-\frac{1}{\mathcal{R}} \frac{\partial}{\partial \mathcal{R}}\left(\mathcal{R} \frac{\partial}{\partial \mathcal{R}}\right) \phi_{n-1}(\mathcal{R}, z) \\
& \equiv-\nabla_{\mathcal{R}}^{2} \phi_{n-1}
\end{aligned}
$$

for $n \geq 1$ subject to $\phi_{n}(\mathcal{R}, z= \pm L / 2)=0$. The unique solution to Eq. (A4) is

$$
\phi_{0}(\mathcal{R}, z)=\frac{L^{2} \tilde{\rho}_{b}(\mathcal{R})}{8 \epsilon_{0}}\left(1-4 \frac{z^{2}}{L^{2}}\right)
$$

and the unique solution to Eq. (A5) is

$$
\begin{aligned}
\phi_{n}(\mathcal{R}, z)= & -\int_{-L / 2}^{z} d \tilde{z} \int_{-L / 2}^{z} d \tilde{\tilde{z}} \nabla_{\mathcal{R}}^{2} \phi_{n-1}(\mathcal{R}, \tilde{\tilde{z}}) \\
& +\frac{z+L / 2}{L} \int_{-L / 2}^{L / 2} d z \int_{-L / 2}^{z} d \tilde{z} \nabla_{\mathcal{R}}^{2} \phi_{n-1}(\mathcal{R}, \tilde{\tilde{z}}) .
\end{aligned}
$$

Successive orders of the expansion for $\phi_{g}$ given by Eq. (A3) and with the $\phi_{n}$ given by Eqs. (A6) and (A7) are well behaved and $\Theta\left(\epsilon^{2}\right)$ smaller than the next-lowest order term for smooth $\rho_{b}(r)$. Using Eq. (A1), the approximate large aspect-ratio solution for $\phi_{g}$ in Eq. (A6) is the leading-order expansion $\phi_{g} \simeq \phi_{0}$.

We note that there is an alternative way to see that Eq. (A6) is the leading-order solution in the limit of small foil spacing: we can use the method of manufactured sources to generate a $z$-dependent correction $\tilde{\rho}$ to $\rho$ such that our $\phi_{0}$ is the exact solution to Poisson's equation. It is easily verified that $\tilde{\rho}$ is $\mathcal{O}\left(\epsilon^{2}\right)$ smaller than $\rho$. 


\section{APPENDIX B: LARMOR-FRAME TRANSFORMATION AND SYSTEM CANONICAL ANGULAR MOMENTUM AND EMITTANCE}

Here, we present a formulation which enables application of the envelope model in Sec. IV to situations where there is an applied solenoidal field $\left(B_{z 0} \neq 0\right)$ which results in a macroscopic rotation of the beam about the $z$ axis. In this case, the formulation presented in Sec. IV, which is valid in laboratory-frame variables as directly expressed when $B_{z 0}=0$, still applies when interpreted "Larmorframe" variables in a frame of reference rotating about the $z$ axis. This generalization requires that the laboratoryframe emittance in Eq. (34) be replaced as specified in Eq. (36). In cases where the beam canonical angular momentum is nonzero (which commonly occurs when the beam is produced in an applied solenoidal field; see Appendix C), this can complicate the proper form of the emittance to employ. In this Appendix, we summarize steps involved in the Larmor transformation, prove that the system canonical angular momentum is invariant, and show how the canonical angular momentum and Larmorframe emittance are related to familiar laboratory-frame quantities. Formulas for the canonical angular momentum and beam emittance which are applicable to common situations in beam transport are derived in Appendix C. More details on these topics can be found in Appendix A of Ref. [36] and Refs. [28,50].

First, the Larmor transform to a rotating (tilde variables) frame is given by[28,36]

$$
\begin{aligned}
& \tilde{x}=x \cos \tilde{\psi}(z)+y \sin \tilde{\psi}(z), \\
& \tilde{y}=-x \sin \tilde{\psi}(z)+y \cos \tilde{\psi}(z)
\end{aligned}
$$

with

$$
\tilde{\psi}(z)=-\frac{q}{2 \gamma_{b} \beta_{b} m c} \int_{z_{i}}^{z} d \tilde{z} B_{z 0}(\tilde{z}) .
$$

This transformation can be applied to show that the equation of motion (30) in $x-y$ laboratory-frame variables becomes

$$
\begin{gathered}
\tilde{\mathbf{x}}_{\perp}^{\prime \prime}+\kappa \tilde{\mathbf{x}}_{\perp}-\left(1-\mathcal{F}_{J}\right) \frac{q}{m \gamma_{b} c^{2}} \frac{\partial \phi_{v}}{\partial \tilde{\mathbf{x}}_{\perp}} \\
\simeq-\left(1-\mathcal{F}_{\rho}\right) \frac{q}{m \gamma_{b} \beta_{b}^{2} c^{2}} \frac{\partial \phi_{g}}{\partial \tilde{\mathbf{x}}_{\perp}}
\end{gathered}
$$

in $\tilde{x}-\tilde{y}$ Larmor-frame variables with $\kappa$ defined in Eq. (32). Here, we have assumed that both $\phi_{v}$ and $\phi_{g}$ are functions only of $r$ and have employed the fact that the Larmor transform (B1) preserves radial distance, i.e., $r=$ $\sqrt{x^{2}+y^{2}}=\sqrt{\tilde{x}^{2}+\tilde{y}^{2}}$. Equation (31) is Eq. (B3) with the tildes dropped (Larmor-frame variables are implicit in Sec. IV when $B_{z 0} \neq 0$ ).

The canonical angular momentum of the beam is calculated by averaging the single-particle canonical angular momentum $p_{\theta}=\tilde{x} \tilde{y}^{\prime}-\tilde{y} \tilde{x}^{\prime}$ over the beam distribution to obtain

$$
\begin{aligned}
P_{\theta} & \equiv\left\langle\tilde{x} \tilde{y}^{\prime}-\tilde{y} \tilde{x}^{\prime}\right\rangle_{\perp} \\
& =\left\langle x y^{\prime}-y x^{\prime}\right\rangle_{\perp}+\frac{q B_{z 0}}{2 m \gamma_{b} \beta_{b} c}\left\langle x^{2}+y^{2}\right\rangle_{\perp} .
\end{aligned}
$$

Here, in the second expression for $P_{\theta}$, the Larmor transformation (B1) and its $z$ derivative has been applied to express $P_{\theta}$ in laboratory-frame variables. Because this is the form of the system angular momentum one would employ in the laboratory frame, we do not use tildes in the symbol for $P_{\theta}$. Differentiating the second expression for $P_{\theta}$ in Eq. (B4) with respect to $z$, applying the laboratory-frame equation of motion (30), and the applied field model in Eq. (1) for $\mathbf{B}^{a}$ shows that

$$
\begin{aligned}
\frac{d}{d z} P_{\theta}= & \frac{q}{m \gamma_{b} c^{2}}\left(1-\mathcal{F}_{J}\right)\left[\left\langle x \frac{\partial \phi_{v}}{\partial y}\right\rangle_{\perp}-\left\langle y \frac{\partial \phi_{v}}{\partial x}\right\rangle_{\perp}\right] \\
& -\frac{q}{m \gamma_{b} \beta_{b}^{2} c^{2}}\left(1-\mathcal{F}_{\rho}\right)\left[\left\langle x \frac{\partial \phi_{g}}{\partial y}\right\rangle_{\perp}-\left\langle y \frac{\partial \phi_{g}}{\partial x}\right\rangle_{\perp}\right]=0 .
\end{aligned}
$$

The second line follows immediately because $\phi_{j}$ with $j=v, g$ is a function of $r$ in the axisymmetric system. Thus, $P_{\theta}=$ const regardless of the functional form of the applied solenoidal field $B_{z 0}(z)$ insofar as the system is axisymmetric. See Appendix $C$ for a derivation of acceleration-induced changes in $P_{\theta}$.

The Larmor-frame rms emittance is explicitly

$$
\tilde{\varepsilon}_{\tilde{x}, \mathrm{rms}} \equiv\left[\left\langle\tilde{x}^{2}\right\rangle_{\perp}\left\langle\tilde{x}^{\prime 2}\right\rangle_{\perp}-\left\langle\tilde{x} \tilde{x}^{\prime}\right\rangle_{\perp}^{2}\right]^{1 / 2} .
$$

Equation (34) is simply Eq. (B6) with the tildes dropped for notational convenience and correspondence to familiar forms in the absence of a solenoidal magnetic field (i.e., $B_{z 0}=0$ and $\mathbf{x}_{\perp}=\tilde{\mathbf{x}}_{\perp}$ ). Use of the Larmor transformation (B1) and its $z$ derivative shows that Eq. (B6) can be expressed in the laboratory frame as

$$
\tilde{\varepsilon}_{\tilde{x}, \mathrm{rms}}^{2}=\varepsilon_{x, \mathrm{rms}}^{2}-\frac{1}{4}\left\langle r^{2} \theta^{\prime}\right\rangle_{\perp}^{2}+\frac{1}{4} P_{\theta}^{2} .
$$

Here, $\theta=\tan ^{-1}(y, x)$, and

$$
\varepsilon_{x, \mathrm{rms}} \equiv\left[\left\langle x^{2}\right\rangle_{\perp}\left\langle x^{\prime 2}\right\rangle_{\perp}-\left\langle x x^{\prime}\right\rangle_{\perp}^{2}\right]^{1 / 2}
$$

denotes the usual, laboratory-frame rms emittance [not to be confused with Eq. (34) which is Eq. (B6) with the tildes dropped]. It is useful in solenoidal focusing systems to denote a beam radial $\mathrm{rms}$ emittance by

$$
\varepsilon_{r, \mathrm{rms}}^{2} \equiv \varepsilon_{x, \mathrm{rms}}^{2}-\frac{1}{4}\left\langle r^{2} \theta^{\prime}\right\rangle_{\perp}^{2} .
$$

Because $P_{\theta}=$ const, if $\tilde{\varepsilon}_{\tilde{x} \text {,rms }}=$ const then Eq. (B7) implies that $\varepsilon_{r, \mathrm{rms}}=$ const. Some straightforward manipulations show that

$$
\left\langle r^{2} \theta^{\prime}\right\rangle_{\perp}=\left\langle r\left\langle r \theta^{\prime}\right\rangle_{\mathbf{p}}\right\rangle_{\perp}
$$

where 


$$
\langle\cdots\rangle_{\mathbf{p}} \equiv \frac{\int d^{3} p \cdots f_{b}}{\int d^{3} p f_{b}}
$$

denotes a restricted momentum (fluid model type) average. Note that $\beta_{b} c\left\langle r \theta^{\prime}\right\rangle_{\mathbf{p}}=V_{\theta}$, where $V_{\theta}$ denotes the transverse azimuthal component of the fluid flow velocity, which can vary in $r$ for the axisymmetric beam. In this context, the term $\propto\left\langle r^{2} \theta^{\prime}\right\rangle_{\perp}^{2}$ subtracted from $\varepsilon_{x, \text { rms }}^{2}$ in Eq. (B9) can be understood as appropriate to remove the azimuthal "fluid" flow of the beam from the transverse phase-space area measure when the beam is macroscopically rotating about the $z$ axis due to the presence of a solenoidal magnetic field $\left(B_{z 0} \neq 0\right)$ and/or finite system canonical angular momentum $\left(P_{\theta} \neq 0\right)$. If $B_{z 0}=0$ and $P_{\theta}=0$, then $\tilde{\varepsilon}_{\tilde{x}, \mathrm{rms}}=$ $\varepsilon_{r, \mathrm{rms}}=\varepsilon_{x, \mathrm{rms}}$ and the envelope formulation in Sec. IV B can be applied with Eq. (34) with the emittance simply interpreted in the laboratory frame. (This is the case for the illustrative example in Sec. V.) However, if $B_{z 0} \neq 0$ and/or $P_{\theta}=0$ the emittance should be replaced with the form in Eq. (36) for correct correspondence to the actual phasespace area. This distinction can be particularly important for beams formed in an axial magnetic field (i.e., source located where $B_{z 0} \neq 0$ ).

\section{APPENDIX C: ESTIMATES OF BEAM EMITTANCE AND CANONICAL ANGULAR MOMENTUM}

It is useful to have explicit formulas for the Larmorframe rms emittance $\tilde{\varepsilon}_{\tilde{x} \text {,rms }}$ [Eq. (B6)] and the beam canonical angular momentum $P_{\theta}$ [Eq. (B4)] which are carefully defined in Appendix B and occur in the statistical envelope equations derived in Sec. IV. Here we provide formulas for these quantities which are applicable to typical laboratory situations and are expressed in terms of commonly measured quantities defined in the laboratory frame.

First, if the axisymmetric beam is incident on the foil lens from an axisymmetric accelerator or transport system where the beam is injected from a source not immersed in an applied axial magnetic field $\left(B_{z 0}=0\right)$, then one expects zero beam canonical angular momentum $P_{\theta}=0$. Conversely, if the beam is born off a source which is immersed in an axial magnetic field $\left(B_{z 0} \neq 0\right)$ and one takes the reasonable boundary condition that the beam is not macroscopically rotating at the emission plane, then Eq. (B4) gives

$$
P_{\theta}=\left.\frac{q B_{z 0}}{m \gamma_{b} \beta_{b} c}\left\langle x^{2}\right\rangle_{\perp}\right|_{z=z_{i}} .
$$

Here, $z=z_{i}$ denotes the axial location of the source. For initial beams with Gaussian- and uniform-charge-density profiles [see Eq. (6)], this gives

$$
P_{\theta}= \begin{cases}\left.\frac{B_{z 0} \sigma_{x}^{2}}{[B \rho]}\right|_{z=z_{i}}, & \text { Gaussian } \\ \left.\frac{B_{z 0} r_{b}^{2}}{4[B \rho]}\right|_{z=z_{i}}, & \text { uniform. }\end{cases}
$$

Here, $[B \rho]=m \gamma_{b} \beta_{b} c / q$ is the particle rigidity. Because $P_{\theta}=$ const, this initial value of $P_{\theta}$ applies for all $z$ for a coasting beam with $\left(\gamma_{b} \beta_{b}\right)=$ const. If the beam is accelerating in the transport lattice upstream of the foil lens with $\left(\gamma_{b} \beta_{b}\right) \neq$ const varying in $z$, then adding the appropriate damping term $-\left[\left(\gamma_{b} \beta_{b}\right)^{\prime} /\left(\gamma_{b} \beta_{b}\right)\right] \tilde{x}^{\prime}$ to the left-hand side of the Larmor-frame equation of motion (31) [28] and paralleling the analysis in Appendix B to calculate $\frac{d P_{\theta}}{d z}$ [see Eq. (B5)] shows that

$$
\frac{d}{d z} P_{\theta}=-\frac{\left(\gamma_{b} \beta_{b}\right)^{\prime}}{\left(\gamma_{b} \beta_{b}\right)} P_{\theta}
$$

Equation (C3) can be integrated with respect to $z$ to show for the accelerating beam that

$$
\gamma_{b} \beta_{b} P_{\theta}=\left.\gamma_{b} \beta_{b} P_{\theta}\right|_{z=z_{i}} .
$$

This result is analogous to the conservation of normalized emittance (see below and Appendix A of Ref. [32]) but is more stringent since it does not require linear dynamics for validity. If the beam is accelerated from a surface immersed in a magnetic field at $z=z_{i}$ where $B_{z 0} \neq 0$, Eqs. (C1) and (C4) show that

$$
P_{\theta}=\frac{1}{m \gamma_{b} \beta_{b} c}\left[q B_{z 0}\left\langle x^{2}\right\rangle_{\perp}\right]_{z=z_{i}}
$$

for the nonaccelerating beam in the foil lens. This result can be applied to a beam produced by a short pulse laser illuminated foil immediately "upstream" of a thin-foil lens immersed in a solenoidal field with $B_{z 0} \neq 0$. In this situation, $\left|B_{z 0}\left(z=z_{i}\right)\right|$ will likely be large unless the emitting foil is placed at the null of a cusp-type field which would also likely lead to reduced field strength in the lens.

Next, we analyze the definition in Eq. (B6) for the Larmor-frame emittance $\tilde{\varepsilon}_{\tilde{x}, \text { rms }}$. Because the radial extent of the axisymmetric beam is not altered by the Larmor transformation (B1), we denote $\sigma_{x}=\langle\tilde{x}\rangle_{\perp}^{1 / 2}$ regardless of the distribution (i.e., need not be Gaussian). Then $\sigma_{x}^{\prime}=$ $\left\langle\tilde{x} \tilde{x}^{\prime}\right\rangle_{\perp}^{1 / 2} /\left\langle\tilde{x}^{2}\right\rangle_{\perp}^{1 / 2}$ and the definition of $\tilde{\varepsilon}_{\tilde{x}, \text { rms }}$ can be equivalently expressed as

$$
\tilde{\varepsilon}_{\tilde{x}, r m s}^{2}=\sigma_{x}^{2}\left\langle\left(\tilde{x}^{\prime}-\sigma_{x}^{\prime}\right)^{2}\right\rangle_{\perp} .
$$

Following the notation in Ref. [32], a local kinetic temperature $\tilde{T}_{x}$ can be defined in the Larmor frame as

$$
\tilde{T}_{x}=m \gamma_{b} \beta_{b}^{2} c^{2}\left\langle\left(\tilde{x}^{\prime}-\left\langle\tilde{x}^{\prime}\right\rangle_{\mathbf{p}}\right)^{2}\right\rangle_{\mathbf{p}}
$$

Here, $\langle\cdots\rangle_{\mathbf{p}}$ is the restricted momentum average defined in Eq. (B11) and the choice in $\gamma_{b}$ factors is motivated in Refs. [32,37]. Note that $\tilde{T}_{x}$ can vary with $r$ in the axisymmetric beam and $\tilde{x}^{\prime}-\left\langle\tilde{x}^{\prime}\right\rangle_{\mathbf{p}}$ is a measure of the difference in the local particle angle from the average (fluid flow) angle 
of all particles in the beam distribution. A spatially average temperature $\overline{\tilde{T}_{x}}$ is then naturally defined by

$$
\frac{\overline{\tilde{T}_{x}}}{m \gamma_{b} \beta_{b}^{2} c^{2}} \equiv \frac{\int d^{2} x \tilde{T}_{x} n_{b}}{\int d^{2} x n_{b}}=\left\langle\left(\tilde{x}^{\prime}-\left\langle\tilde{x}^{\prime}\right\rangle_{\mathbf{p}}\right)^{2}\right\rangle_{\perp} .
$$

Here, $n_{b} \equiv \int d^{3} p f_{b}$ denotes the beam density, and $\left\langle\langle\cdots\rangle_{\mathbf{p}}\right\rangle_{\perp}=\langle\cdots\rangle_{\perp}$ has been employed. We approximate

$$
\left\langle\tilde{x}^{\prime}\right\rangle_{\mathbf{p}} \simeq \sigma_{x}^{\prime} \frac{\tilde{x}}{\sigma_{x}},
$$

which effectively replaces the local coherent angle $\left\langle\tilde{x}^{\prime}\right\rangle_{\mathbf{p}}$ of the distribution by the form appropriate for an rmsequivalent Kapchinskij-Vladimirskij (KV) beam distribution (see Refs. $[28,32]$ ). Then it is straightforward to show using Eqs. (C8) and (C9) that

$$
\frac{\overline{\tilde{T}_{x}}}{m \gamma_{b} \beta_{b}^{2} c^{2}} \simeq\left\langle\left(\tilde{x}^{\prime}-\sigma_{x}^{\prime}\right)^{2}\right\rangle_{\perp} .
$$

Using this result, Eq. (C6) is approximated as

$$
\tilde{\varepsilon}_{x, \mathrm{rms}}^{2} \simeq \frac{\sigma_{x}^{2} \overline{\tilde{T}_{x}}}{m \gamma_{b} \beta_{b}^{2} c^{2}} .
$$

Equation (C11) can be applied with the spatially average temperature $\tilde{T}_{x}$ as defined in Eq. (C8) to estimate the emittance for an arbitrary distribution (Gaussian, uniform, or any other). If the beam is not macroscopically rotating due to an applied magnetic field, then $\bar{T}_{x}$ can be simply calculated in the laboratory frame (just drop all tildes). If there is acceleration, then arguments presented in Appendix A of Ref. [32] can be applied to show that if "normalized emittance" conservation is assumed (meaning forces acting on the beam are approximated as linear), then the value of emittance at $z$ is related to its initial value by

$$
\gamma_{b} \beta_{b} \tilde{\varepsilon}_{\tilde{x}, \mathrm{rms}}=\left.\gamma_{b} \beta_{b} \tilde{\varepsilon}_{\tilde{x}, \mathrm{rms}}\right|_{z=z_{i}} .
$$

\section{APPENDIX D: DERIVATION OF EQ. (55)}

Denote $\sigma_{x}=\left\langle x^{2}\right\rangle_{\perp}^{1 / 2}$. Here, as in Appendix C, the charge-density profile of the distribution need not be Gaussian and $\sigma_{x}$ is merely employed for notational convenience. Following methods presented in Ref. [36], a conserved envelope Hamiltonian can be derived for Eq. (53) to show that

$$
H=\frac{1}{2} \sigma_{x}^{\prime 2}+Q_{\text {eff }} \ln \sigma_{x}=\text { const. }
$$

Equation (D1) can be set equal to its initial value at $z=z_{i}$ and solved for $\sigma_{x}^{\prime}$ to obtain

$$
\sigma_{x}^{\prime}= \pm \sqrt{\sigma_{x i}^{\prime 2}-2 Q_{\mathrm{eff}} \ln \left(\frac{\sigma_{x}}{\sigma_{x i}}\right)} .
$$

We first integrate Eq. (D2) from an initial condition with $\sigma_{x i}^{\prime} \leq 0$ (converging beam case) which ensures that the minus solution in Eq. (D2) holds for all $z$ to the $\sigma_{x}=0$ focal point. This obtains

$$
\begin{aligned}
z-z_{i}= & \sqrt{\frac{\pi}{2 Q_{\text {eff }}}} \exp \left(\frac{\sigma_{x i}^{\prime 2}}{2 Q_{\text {eff }}}\right) \sigma_{x i}\left\{\operatorname{erf}\left[\sqrt{\frac{\sigma_{x i}^{\prime 2}}{2 Q_{\text {eff }}}-\ln \left(\frac{\sigma_{x}}{\sigma_{x i}}\right)}\right]\right. \\
& \left.-\operatorname{erf}\left(\sqrt{\frac{\sigma_{x i}^{\prime 2}}{2 Q_{\text {eff }}}}\right)\right\},
\end{aligned}
$$

for $\sigma_{x}>0$. Here, $\operatorname{erf}(z) \equiv \frac{2}{\sqrt{\pi}} \int_{0}^{z} d t e^{-t^{2}}$ is the error function [35]. Evaluating Eq. (D3) at the focal point $z=z_{f}$ where $\sigma_{x} \rightarrow 0$ and employing $\lim _{z \rightarrow \infty} \operatorname{erf}(z)=1$ gives Eq. (55) with $\operatorname{sgn}\left(\sigma_{x i}^{\prime}\right)=-1$.

Next, we integrate Eq. (D2) from an initial condition with $\sigma_{x i}^{\prime}>0$ (diverging beam case) by first considering an intermediate axial coordinate $z=z_{t}$ where $\sigma_{x}^{\prime}\left(z=z_{t}\right)=0$ corresponding to where the trajectory to the focal spot is at an inflection point (i.e., $\sigma_{x}^{\prime}=0$ at $z=z_{t}$ ) where the beam is transitioning from diverging to converging. At $z=z_{t}$, Eq. (D2) gives

$$
\sigma_{x}\left(z=z_{t}\right)=\sigma_{x i} \exp \left(\frac{\sigma_{x i}^{\prime 2}}{2 Q_{\text {eff }}}\right) .
$$

Up to the inflection point $\sigma_{x}^{\prime}>0$, the plus root in Eq. (D2) applies. Analogous steps leading to Eqs. (D3) and (55) in the case with $\sigma_{x i}^{\prime} \leq 0$ together with an application of Eq. (D4) then give

$$
z_{t}-z_{i}=\sqrt{\frac{\pi}{2 Q_{\text {eff }}}} \exp \left(\frac{\sigma_{x i}^{\prime 2}}{2 Q_{\text {eff }}}\right) \sigma_{x i} \operatorname{erf}\left(\sqrt{\frac{\sigma_{x i}^{\prime 2}}{2 Q_{\text {eff }}}}\right)
$$

for the distance of the diverging interval where $\sigma_{x}^{\prime} \geq 0$. The subsequent converging interval from the inflection point to the focal pinch has $\sigma_{x}^{\prime} \leq 0$ and can then be obtained from Eq. (D3) in the already derived case with $\sigma_{x i}^{\prime} \leq 0$ by substituting $z_{i} \rightarrow z_{t}, \quad \sigma_{x i} \rightarrow \sigma_{x}\left(z=z_{t}\right)=$ $\sigma_{x i} \exp \left[\sigma_{x i}^{\prime 2} /\left(2 Q_{\text {eff }}\right)\right]$, and $\sigma_{x i}^{\prime} \rightarrow 0$ to show that

$$
z_{f}-z_{t}=\sqrt{\frac{\pi}{2 Q_{\mathrm{eff}}}} \exp \left(\frac{\sigma_{x i}^{\prime 2}}{2 Q_{\mathrm{eff}}}\right) \sigma_{x i} .
$$

Adding Eqs. (D5) and (D6) gives Eq. (55) with $\operatorname{sgn}\left(\sigma_{x i}^{\prime}\right)=+1$ to complete the derivation.

[1] M. Roth, A. Blazevic, M. Geissel, T. Schlegel, T.E. Cowan, M. Allen, J.-C. Gauthier, P. Audebert, J. Fuchs, J.M. ter Vehn et al., Phys. Rev. ST Accel. Beams 5, 061301 (2002).

[2] M. Roth et al., Plasma Phys. Controlled Fusion 51, 124039 (2009), and references therein.

[3] M. Roth, T.E. Cowan, M.H. Key, S. P. Hatchett, C. Brown, W. Fountain, J. Johnson, D. M. Pennington, R. A. Snavely, S. C. Wilks et al., Phys. Rev. Lett. 86, 436 (2001). 
[4] P. K. Patel, A. J. Mackinnon, M.H. Key, T.E. Cowan, M.E. Foord, M. Allen, D.F. Price, H. Ruhl, P.T. Springer, and R. Stephens, Phys. Rev. Lett. 91, 125004 (2003), and references therein.

[5] T. Bartal et al., Nat. Phys. 8, 139 (2011), and references therein.

[6] E. Henestroza, B. G. Logan, and L. J. Perkins, Phys. Plasmas 18, 032702 (2011).

[7] K. Harres, I. Alber, A. Tauschwitz, V. Bagnoud, H. Daido, M. Gunther, F. Nurnberg, A. Otten, M. Schollmeier, J. Schtrumpf et al., Phys. Plasmas 17, 023107 (2010).

[8] M. Nishiuchi et al., Phys. Rev. ST Accel. Beams 13, 071304 (2010).

[9] R.F. Hubbard, S. A. Goldstein, and D. A. Tidman, Proceedings of the Heavy Ion Fusion Workshop, Berkeley, CA, 1979, p. 488; obtainable by Lawrence Berkeley Laboratory Report No. LBL-10301 and Stanford Linear Accelerator Laboratory Report No. SLAC-R-542.

[10] P. Ni, F. M. Bieniosek, R. Cohen, O. Deppert, B. G. Logan, S. M. Lund, M. Roth, and G. Schaumann (unpublished); see also Lawrence Berkeley Laboratory Report No. UCRL AFFE 107.

[11] M. Roth (private communication).

[12] P. A. Ni, B. G. Logan, S. M. Lund, N. Alexander, F. M. Bieniosek, R.H. Cohen, M. Roth, and G. Schaumann, Laser Part. Beams 31, 81 (2013).

[13] P. Mora, Phys. Rev. Lett. 90, 185002 (2003).

[14] P. Mora, Phys. Rev. E 72, 056401 (2005).

[15] F. Nürnberg, M. Schollmeier, E. Brambrink, A. Blažević, D. C. Carroll, K. Flippo, D. C. Gautier, M. Geissel, K. Harres, B. M. Hegelich et al., Rev. Sci. Instrum. 80, 033301 (2009).

[16] T. Burris-Mog, K. Harres, F. Nürnberg, S. Busold, M. Bussmann, O. Deppert, G. Hoffmeister, M. Joost, M. Sobiella, A. Tauschwitz et al., Phys. Rev. ST Accel. Beams 14, 121301 (2011).

[17] A. Yuen, S. M. Lund, J. J. Barnard, and R. H. Cohen, in the 54rd Annual Meeting of the American Physical Society, Division of Plasma Physics, Providence, RI (2012), abstract number PP8.00146, and a forthcoming article to be submitted.

[18] N. W. Ashcroft and N.D. Mermin, Solid State Physics (W.B. Saunders Company, Philadelphia, 1976).

[19] R. J. Adler, Part. Accel. 12, 39 (1982).

[20] S. Humphries, C. Ekdahl, and D. M. Woodall, Appl. Phys. Lett. 54, 2195 (1989).

[21] R. F. Fernsler, R. F. Hubbard, and S. P. Slinker, J. Appl. Phys. 68, 5985 (1990).

[22] C.L. Olson and U. Schumacher, Collective Ion Acceleration, , Springer Tracts in Modern Physics Vol. 84 (Springer-Verlag, Heidelberg, 1979).

[23] B. G. Logan, Lawrence Berkeley Laboratory seminar (unpublished).

[24] R. A. Snavely et al., Phys. Rev. Lett. 85, 2945 (2000).

[25] S. Hatchett, C. Brown, T. E. Cowan, E. A. Henry, J. Johnson, M. H. Key, J. A. Koch, A. B. Langdon, B. F. Lasinsky, R.W. Lee et al., Phys. Plasmas 7, 2076 (2000).

[26] M. Roth, T. E. Cowan, C. Brown, M. Christl, W. Fountain, S. Hatchett, J. Johnson, M. H. Key, D. M. Pennington,
M. D. Perry et al., Nucl. Instrum. Methods Phys. Res., Sect. A 464, 201 (2001).

[27] M. Borghesi, A. Mackinnon, D. Campbell, D. Hicks, S. Kar, P. Patel, D. Price, L. Romagnani, A. Schiavi, and O. Willi, Phys. Rev. Lett. 92, 055003 (2004).

[28] J. J. Barnard and S. M. Lund, U.S. Particle Accelerator School courses: Lawrence Livermore National Laboratory Report No. LLNL-AR-407617; Lawrence Berkeley National Laboratory Report No. LBNL1097E; Lawrence Livermore National Laboratory Report No. UCRL-TM-231628; Lawrence Berkeley National Laboratory Report No. LBNL-62783; Lawrence Livermore National Laboratory Report No. UCRL-TM-203655; Lawrence Berkeley National Laboratory Report No. LBNL-54926; Lawrence Berkeley National Laboratory Report No. LBNL49286.

[29] S. M. Lund, C. J. Wootton, and E. P. Lee, Nucl. Instrum. Methods Phys. Res., Sect. A 606, 56 (2009).

[30] E. Brambrink, M. Roth, A. Blazevic, and T. Schlegel, Laser Part. Beams 24, 163 (2006).

[31] M. Schollmeier, K. Harres, F. Nurnberg, A. Blazevic, P. Audebert, E. Brambrink, J.C. Fernandez, K. A. Flippo, D. C. Gautier, M. Geissel et al., Phys. Plasmas 15, 053101 (2008).

[32] S. M. Lund, T. Kikuchi, and R. C. Davidson, Phys. Rev. ST Accel. Beams 12, 114801 (2009).

[33] S. M. Lund, A. Friedman, and G. Bazouin, Phys. Rev. ST Accel. Beams 14, 054201 (2011).

[34] J.D. Jackson, Classical Electrodynamics (John Wiley \& Sons, New York, 1975), 2nd ed.

[35] Handbook of Mathematical Functions with Formulas, Graphs, and Mathematical Tables, edited by M. Abramowitz and I. A. Stegun (Dover, New York, 1972), 9th ed.

[36] S. M. Lund and B. Bukh, Phys. Rev. ST Accel. Beams 7, 024801 (2004).

[37] M. Reiser, Theory and Design of Charged Particle Beams (John Wiley \& Sons, New York, 1994).

[38] F. J. Sacherer, Ph.D. thesis, University of California, 1968.

[39] F. J. Sacherer, IEEE Trans. Nucl. Sci. 18, 1105 (1971).

[40] B. M. Hegelich, B. J. Albright, J. Cobble, K. Flippo, S. Letzring, M. Paffett, H. Ruhl, J. Schreiber, R. K. Schulze, and J.C. Fernández, Nature (London) 439, 441 (2006).

[41] H. Schwoerer, S. Pfotenhauer, O. Jäckel1, K.-U. Amthor, B. Liesfeld, W. Ziegler, R. Sauerbrey, K.W.D. Ledingham, and T. Esirkepov, Nature (London) 439, 445 (2006).

[42] S. Ter-Avetisyan, M. Schnuürer, P. V. Nickles, M. Kalashnikov, E. Risse, T. Sokollik, W. Sandner, A. Andreev, and V. Tikhonchuk, Phys. Rev. Lett. 96, 145006 (2006).

[43] X. Q. Yan, C. Lin, Z. M. Sheng, Z. Y. Guo, B. C. Liu, Y. R. Lu, J.X. Fang, and J.E. Chen, Phys. Rev. Lett. 100, 135003 (2008).

[44] A. Henig, S. Steinke, M. Schnürer, T. Sokollik, R. Hörlein, D. Kiefer, D. Jung, J. Schreiber, B. M. Hegelich, X. Q. Yan et al., Phys. Rev. Lett. 103, 245003 (2009). 
[45] R. Sonobe, S. Kawata, S. Miyazaki, M. Nakamura, and T. Kikuchi, Phys. Plasmas 12, 073104 (2005).

[46] M. Nakamura, S. Kawata, R. Sonobe, Q. Kong, S. Miyazaki, N. Onuma, and T. Kikuchi, J. Appl. Phys. 101, 113305 (2007).

[47] D. P. Grote, Ph.D. thesis, University of California at Davis, 1994.
[48] D. P. Grote, A. Friedman, I. Haber, W. Fawley, and J.-L. Vay, Nucl. Instrum. Methods Phys. Res., Sect. A 415, 428 (1998).

[49] D. P. Grote, E. Henestroza, and J. W. Kwan, Phys. Rev. ST Accel. Beams 6, 014202 (2003).

[50] H. Wiedemann, Particle Accelerator Physics II: Nonlinear and Higher-Order Beam Dynamics (Springer-Verlag, New York, 2004), 2nd ed. 